\title{
The Potential Constants of Ethane
}

\author{
G. E. HANSEN* $\dagger$ AND D. M. DENNiSON \\ Randall Laboratory of Physics, University of Michigan, Ann Arbor, Michigan
}

(Received August 3, 1951)

The infrared and Raman data of light and heavy ethane $\left(\mathrm{C}_{2} \mathrm{H}_{6}\right.$ and $\mathrm{C}_{2} \mathrm{D}_{6}$ ) have been reexamined for the purpose of determining as accurately as possible the potential constants of the ethane molecule. In order to fill in some of the gaps in the spectroscopic data, additional high resolution measurements have been made on the infrared spectrum of heavy ethane which have given more precise values for the active fundamental frequencies and zetavalues. Resolution of the fine structure associated with the parallel band $\nu_{5}{ }^{*}$ has given the value of the large moment of inertia of $\mathrm{C}_{2} \mathrm{D}_{6}$, thus completing the information required for the spectroscopic determination of the dimensions of ethane. The data yield, $\mathrm{C}-\mathrm{C}$ distance $=1.543 \mathrm{~A}, \mathrm{C}-\mathrm{H}$ distance $=1.102 \mathrm{~A}, \mathrm{H}-\mathrm{C}-\mathrm{C}$ angle $=109^{\circ} 37^{\prime}$, and $\mathrm{H}-\mathrm{C}-\mathrm{H}$ angle $=109^{\circ} 19^{\prime}$. The twenty-two distinct potential constants compatible with the $\mathrm{D}_{3 d}$ symmetry of ethane have been determined through their relationships to the normal frequencies and zeta-values of $\mathrm{C}_{2} \mathrm{H}_{6}$ and $\mathrm{C}_{2} \mathrm{D}_{6}$. The normal frequencies have been obtained by addition of anharmonic corrections to the spectroscopically observed fundamental frequencies. These corrections were estimated by means of the known anharmonic corrections for methane and the conditions imposed by the Teller product rule. The fundamental frequencies and zeta-values have been taken directly from the observed band centers and rotational spacings wherever possible. In the cases of resonance, the influence of the couplings were either calculated or estimated and the corresponding unperturbed values for the frequencies and zeta-values selected. The potential function is determined first in terms of a set of simple symmetry coordinates, and then reexpressed in terms of valence coordinates to permit comparison of the valence force constants of ethane and methane.

\section{INTRODUCTION}

$S^{\mathrm{H}}$ HORTLY after the initial measurements of the ethane spectrum, ${ }^{1}$ analyses of the observed fundamental frequencies led to the construction of simplified potential functions ${ }^{2}$ which not only indicated the magnitudes of the principal restoring forces but also aided in the later assignments of additional fundamental and overtone bands. By 1938, Crawford, Avery, and Linnet $\mathrm{t}^{3}$ were able to give a complete summary of the twelve distinct fundamental frequencies although, as they emphasized, the spectroscopic data were insufficient to determine whether the equilibrium configuration of the molecule is the "staggered" model $\mathrm{D}_{3 d}$ (center of symmetry) or the "eclipsed" model $\mathrm{D}_{3 h}$ (reflection plane perpendicular to the symmetry axis).

In 1939 Stitt $^{4}$ measured the infrared and Raman spectra of $\mathrm{C}_{2} \mathrm{D}_{6}$, and determined a set of potential constants for ethane, correlating the data for these two isotopic molecules and illustrating the importance of the forces coupling different bond distortions. Recently, the high resolution measurements by Smith ${ }^{5}$ considerably improved and extended the infrared data for ethane. The observed combination bands gave direct evidence for the $\mathrm{D}_{3 d}$ equilibrium configuration while the rotational spacings gave the moments of inertia for this molecule as well as the zeta-values associated with the degenerate vibrations.

The purpose of the present investigation is the re-

* Based on a dissertation submitted to the Graduate School of the University of Michigan in partial fulfilment of the requirements for the degree of Doctor of Philosophy.

$\dagger$ Present address: Los Alamos Scientific Laboratory, Los Alamos, New Mexico.

1 A. Levin and C. F. Meyer, J. Opt. Soc. Am. 16, 137 (1928); P. Daure, Ann. phys. 12, 375 (1929).

${ }^{2}$ G. Sutherland and D. M. Dennison, Proc. Roy. Soc. (London) A148, 250 (1935) ; J. B. Howard, J. Chem. Phys. 5, 442 (1937).

${ }^{3}$ Crawford, Avery, and Linnett, J. Chem. Phys. 6, 682 (1938).

${ }^{4}$ F. Stitt, J. Chem. Phys. 7, 297 (1939).

5 L. G. Smith, J. Chem. Phys. 17, 139 (1949). evaluation of the present accumulation of spectroscopic data, the filling in of some of the gaps by additional measurements of the infrared spectrum of $\mathrm{C}_{2} \mathrm{D}_{6}$, and the determination of a set of potential constants for ethane which best describes this information.

At the present time, complete sets of potential constants have been determined for only a few of the simpler polyatomic molecules such as $\mathrm{CO}_{2}, \mathrm{H}_{2} \mathrm{O}, \mathrm{NH}_{3}$, and $\mathrm{CH}_{4}{ }^{6}$ There are two inherent difficulties involved in the calculation, the first of which is that the potential constants are related to the normal vibrational frequencies of the molecule. The observed spectrum, however, yields the fundamental frequencies and these differ from the normal frequencies by amounts of the order of a few percent. These differences are caused principally by the higher order terms, cubic and quartic, in the potential energy and can only be evaluated when all of the overtones involving pairs of fundamental as well as harmonics of fundamental frequencies have been observed. In the case of ethane with its 12 fundamentals, this would require the identification and measurement of 114 overtones and harmonics. This large amount of information is not available, and consequently other methods have been employed which appear to be reliable at least as first approximations. These methods consist in the use of the product rule and the frequencies of such harmonics as are known, together with a plausible assumption regarding the ratio of the anharmonicity factor in isotopic molecules as will be described in Section III.

The second difficulty arises from the fact that while the normal frequencies are functions of the potential constants, there are in general more constants than frequencies. Thus, if a molecule possess $n$ normal vibrations belonging to a particular symmetry class, there will be $\frac{1}{2} n(n+1)$ constants in the general potential

${ }^{6}$ D. M. Dennison, Revs. Modern Phys. 12, 175 (1940). 


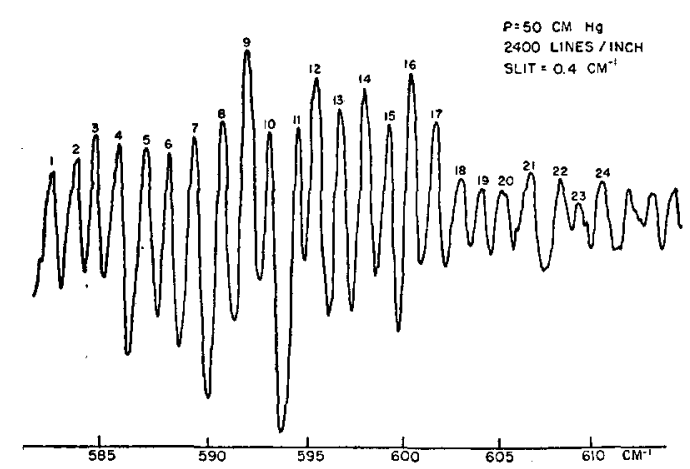

FIG. 1. Absorption by the fundamental $\nu_{9}^{*}=593.7 \mathrm{~cm}^{-1}$. Lines 9,12 , and 16 are enhanced by the presence of water vapor in the absorption path. The rotational spacing $\Delta \nu_{9}{ }^{*}=1.197 \mathrm{~cm}^{-1}$ corresponds to the zeta-value $\zeta_{9}^{*}=0.182$.

function. There are a number of ways out of this difficulty of which the one which has been most widely used has been to approximate the form of the potential function, for example, by assuming valence type forces, and thus reduce the number of unknown constants. A much more satisfactory method, whenever the experimental data are available, is to employ (1) the normal frequencies of isotopic molecules and (2) the zeta-values. These latter measure the internal angular momenta associated with the degenerate vibrations and are also functions of the potential constants. The data on ethane are at present sufficient to make this method possible in all but one of the symmetry classes. The situation is as follows:

\section{A. Perpendicular Active Vibrations, $E_{u}$}

There are three frequencies $\omega_{7}, \omega_{8}$, and $\omega_{9}$ belonging to this class, and hence six potential constants. The normal frequencies for both light and heavy ethane have been determined as well as the three zetas for light ethane and two of the heavy ethane zetas. The sum of the zetas for any symmetry class is fixed, however, and the product rule imposes a relation between the light and heavy normal frequencies. There are thus nine independent data with which to determine the six

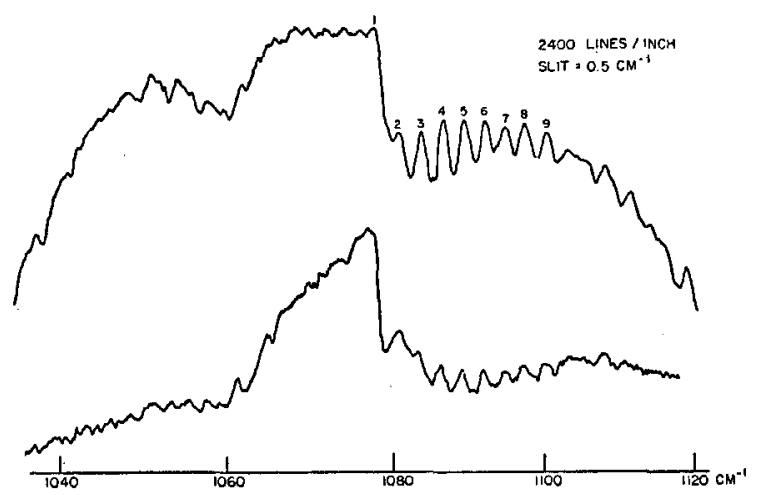

FIG. 2. Absorption by the fundamentals $\nu_{6}{ }^{*} \cong 1077 \mathrm{~cm}^{-1}$ and $\nu_{8}^{*} \cong 1082 \mathrm{~cm}^{-1}$. The rotational spacing $\Delta \nu_{8}^{*}=2.76 \mathrm{~cm}^{-1}$ corresponds to the zeta-value $\zeta_{8}^{*}=-0.423$.
TABLE I. Wave numbers of the lines shown in Fig. 1.

\begin{tabular}{crrr}
\hline \hline Line no. & \multicolumn{2}{c}{$K^{\prime \prime} \rightarrow K^{\prime}$} & $\mathrm{cm}^{-1}$ \\
\hline 1 & 10 & 9 & 582.3 \\
2 & 9 & 8 & 583.6 \\
3 & 8 & 7 & 584.7 \\
4 & 7 & 6 & 585.9 \\
5 & 6 & 5 & 587.1 \\
6 & 5 & 4 & 588.1 \\
7 & 4 & 3 & 589.3 \\
8 & 3 & 2 & 590.6 \\
9 & 2 & 1 & 591.8 \\
10 & 1 & 0 & 593.0 \\
11 & 0 & 1 & 594.3 \\
12 & 1 & 2 & 595.3 \\
13 & 2 & 3 & 596.5 \\
14 & 3 & 4 & 597.7 \\
15 & 4 & 5 & 598.9 \\
16 & 5 & 6 & 600.2 \\
17 & 6 & 7 & 601.3 \\
18 & 7 & 8 & 602.5 \\
19 & 8 & 9 & 603.8 \\
20 & 9 & 10 & 605.1 \\
21 & 10 & 11 & 606.4 \\
22 & 11 & 12 & 607.8 \\
23 & 12 & 13 & 609.1 \\
24 & 13 & 14 & 610.4 \\
\hline
\end{tabular}

constants. A least squares solution gives a very satisfactory fit.

\section{B. Perpendicular Inactive Vibrations, $E_{g}$}

The three normal frequencies $\omega_{10}, \omega_{11}$, and $\omega_{12}$ are again known for both the light and heavy ethane and two of the light zetas have been measured. These constitute seven independent data and the six potential constants of this symmetry class may be found.

\section{Parallel Inactive Vibrations, $\mathbf{A}_{1 \sigma}$}

The three normal frequencies $\omega_{1}, \omega_{2}$, and $\omega_{3}$ have been found for both the isotopic molecules but, because of the product rule, these constitute only five data. Since the vibrations are not degenerate, there exist no zetas. The six potential constants cannot be calculated without employing a further assumption, and the one that has been made is that the cross product term between the $\mathrm{C}-\mathrm{C}$ and the $\mathrm{C}-\mathrm{H}$ stretchings has been taken to be zero.

\section{Parallel Active Vibrations, $A_{2 u}$}

Two frequencies $\omega_{6}$ and $\omega_{6}$ belong to this class and have been measured in both light and heavy ethane.

TABLE II. Wave numbers of the lines shown in Fig. 2.

\begin{tabular}{cccc}
\hline \hline Line no. & \multicolumn{2}{c}{$J^{\prime \prime} \rightarrow J^{\prime}$} & $\mathrm{cm}^{-1}$ \\
\hline 1 & 0 & 0 & 1077.1 \\
& \multicolumn{2}{c}{$K^{\prime \prime} \rightarrow K^{\prime}$} & \\
2 & 1 & 0 & 1080.4 \\
3 & 0 & 1 & 1083.2 \\
4 & 1 & 2 & 1086.2 \\
5 & 2 & 3 & 1089.0 \\
6 & 3 & 4 & 1091.6 \\
7 & 4 & 5 & 1094.2 \\
8 & 5 & 8 & 1096.9 \\
9 & 6 & 7 & 1099.7 \\
\hline
\end{tabular}


They represent three independent data and hence are just sufficient to find the three potential constants.

\section{E. Torsional Vibration, $A_{1 u}$}

There is only one frequency in this class, namely $\omega_{4}$, and it therefore determines the single potential constant.

In the final section of the paper the potential constants of ethane are expressed in terms of the stretching and bending of the valence bonds and bond angles of the molecule. As might have been expected from previous experience, it is found that the principal contributions come from the stretching of the valence bonds and from the bending of the bond angles, and that the interaction terms between adjacent bonds are, with one or two exceptions, quite small while the interactions between remote bonds are virtually zero.

\section{INFRARED MEASUREMENTS ON $\mathrm{C}_{2} \mathrm{D}_{6}$}

The purpose of remeasuring the infrared spectrum of heavy ethane was to obtain (1) more accurate positions
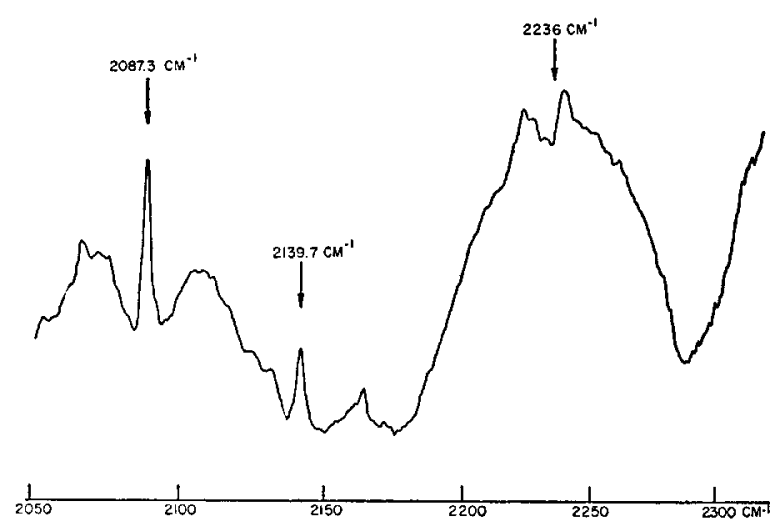

FIG. 3. General record of absorption in the 4.3-5.0 micron region indicating the positions of the parallel doublet bands $\nu_{5 a}{ }^{*}=2087.3$ $\mathrm{cm}^{-1}$ and $\nu_{5 b}{ }^{*}=2139.7 \mathrm{~cm}^{-1}$ and the perpendicular band $\nu_{7}{ }^{*} \cong 2236$ $\mathrm{cm}^{-1}$.

of the band centers associated with the fundamental vibrations, (2) the zeta-values associated with the rotational spacings of the degenerate vibrations, and (3) the large moment of inertia, this being the last parameter required for the spectroscopic determination of the dimensions of ethane.

The measurements were made with a prism-grating spectrometer and automatic recording attachments such as described by W. E. Anderson. ${ }^{7}$ The $\mathrm{C}_{2} \mathrm{D}_{6}$ was prepared by a method similar to that described by $\mathrm{Stitt}^{5}$ (generation and catalytic combination of $\mathrm{D}_{2}$ and $\mathrm{C}_{2} \mathrm{D}_{2}$ utilizing heavy water as the source of deuterium).

Spectrograms of the absorption by the active fundamentals are given in Figs. 1-4, while Tables I-III give the wave number values and assignments of the principal absorption peaks. The notation for any vibration of heavy ethane is the starred symbol for the analogous

\footnotetext{
${ }^{7}$ W. E. Anderson, thesis (University of Michigan, 1948).
}

TABLE III. Wave numbers of the lines shown in Fig. 4.

\begin{tabular}{|c|c|c|c|}
\hline \multirow{4}{*}{ 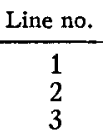 } & \multicolumn{2}{|c|}{$J^{\prime \prime} \rightarrow J^{\prime}$} & \multirow{2}{*}{$\frac{\mathrm{cm}^{-\mathbf{i}}}{2066.88}$} \\
\hline & 23 & 22 & \\
\hline & 22 & 21 & 2067.52 \\
\hline & 21 & 20 & 2068.51 \\
\hline 4 & 20 & 19 & 2069.40 \\
\hline 5 & 19 & 18 & 2070.05 \\
\hline 6 & 18 & 17 & 2071.15 \\
\hline 7 & 17 & 16 & 2072.03 \\
\hline 8 & 16 & 15 & 2072.83 \\
\hline 9 & 15 & 14 & 2073.67 \\
\hline 10 & 14 & 13 & 2074.65 \\
\hline 11 & 13 & 12 & 2075.66 \\
\hline 12 & 12 & 11 & 2076.48 \\
\hline 13 & 11 & 10 & 2077.34 \\
\hline 14 & 10 & 9 & 2078.28 \\
\hline 15 & 9 & 8 & 2079.03 \\
\hline 16 & 8 & 7 & 2080.21 \\
\hline 17 & 7 & 6 & 2080.88 \\
\hline 18 & 6 & 5 & 2082.10 \\
\hline 19 & 5 & 4 & 2082.93 \\
\hline 20 & 0 & 0 & 2087.3 \\
\hline 21 & 7 & 8 & 2094.59 \\
\hline 22 & 8 & 9 & 2095.73 \\
\hline 23 & 9 & 10 & 2096.62 \\
\hline 24 & 10 & 11 & 2097.51 \\
\hline 25 & 11 & 12 & 2098.53 \\
\hline 26 & 12 & 13 & 2099.58 \\
\hline 27 & 13 & 14 & 2100.58 \\
\hline 28 & 14 & 15 & 2101.47 \\
\hline 29 & 15 & 16 & 2102.51 \\
\hline 30 & 16 & 17 & 2103.40 \\
\hline 31 & 17 & 18 & 2104.20 \\
\hline 32 & 18 & 19 & 2105.04 \\
\hline 33 & 19 & 20 & 2105.97 \\
\hline 34 & 20 & 21 & 2107.20 \\
\hline 35 & 21 & 22 & 2108.07 \\
\hline 36 & 22 & 23 & 2109.02 \\
\hline 37 & 23 & 24 & 2109.70 \\
\hline 38 & 24 & 25 & 2111.07 \\
\hline
\end{tabular}

vibration of ethane. Similarly for the other quantities such as zeta-values and moments of inertia.

The two parallel bands shown in Fig. 3 arise from the fundamental vibration $\nu_{5}{ }^{*}$ and a parallel combination band, presumably $\nu_{8+11} *$. Because of the close proximity of the corresponding frequencies, any coupling between these vibrations is of the resonance type and in this case neither of the observed bands may properly be called the fundamental. Evidence of such coupling is furnished by the appreciable intensity of both of these

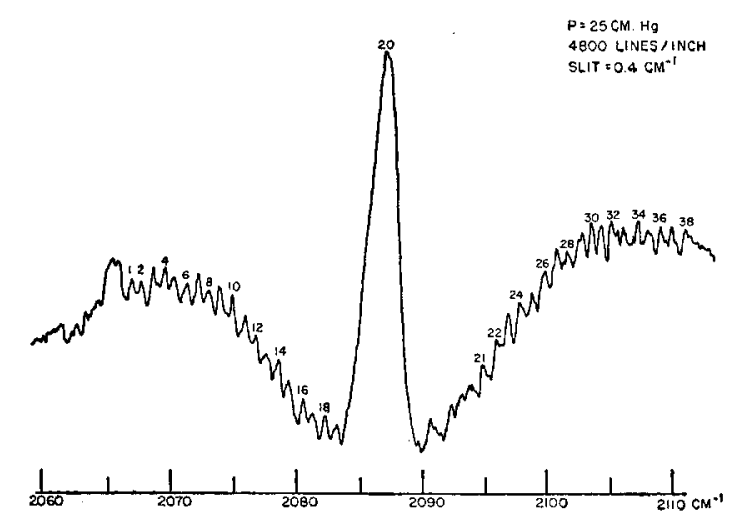

FIG. 4. Fine structure of the fundamental $\nu_{b a}{ }^{*}$. 
bands (and also of the analogous bands of ethane), and consequently they are labelled $\nu_{50}{ }^{*}$ and $\nu_{50}{ }^{*}$.

Figure 4 gives one of the four spectrograms of $\nu_{5 a}{ }^{*}$ recorded under conditions of maximum resolution. For each record the rotational constant $B_{0}^{*}$ for the vibrational ground state was obtained, using the "method of combination differences" suggested by Herzberg. ${ }^{8}$ The resulting mean value, $B_{0}{ }^{*}=0.4610 \pm 0.0010 \mathrm{~cm}^{-1}$, corresponds to a large moment of inertia, $I_{x}^{*}=60.71 \times 10^{-40}$ $\mathrm{g} \mathrm{cm}^{2}$.

Smith's ${ }^{6}$ high resolution measurements of the bands of light ethane have yielded the following values for the large and small moments of inertia of that molecule, $I_{x}=42.234 \times 10^{-40}$ and $I_{z}=10.81 \times 10^{-40} \mathrm{~g} \mathrm{~cm}^{2}$. Since the molecule contains only three independent structural parameters, the above data are sufficient for their determination. One obtains for the distances and angles, $\mathrm{C}-\mathrm{C}=1.543 \times 10^{-8}, \mathrm{C}-\mathrm{H}=1.102 \times 10^{-8} \mathrm{~cm}, \mathrm{H}-\mathrm{C}-\mathrm{C}$ angle $=109^{\circ} 37^{\prime}, \mathrm{H}-\mathrm{C}-\mathrm{H}$ angle $=109^{\circ} 19^{\prime}$. It will be noted that, whereas the angles differ only slightly from the tetrahedral angle of $109^{\circ} 28^{\prime}$, the $\mathrm{C}-\mathrm{H}$ distance is appreciably larger than the $\mathrm{C}-\mathrm{H}$ distance in methane of $1.093 \times 10^{-8} \mathrm{~cm}$. The moments of inertia of both ethane and methane from which the above figures were derived are the moments in the ground state of vibration and not the equilibrium moments, and hence, strictly speaking, they determine effective but not equilibrium dimensions.

In Fig. 2, eight $Q$ lines of the perpendicular band $\nu_{8}{ }^{*}$ are visible between 1080 and $1100 \mathrm{~cm}^{-1}$. The absorption maximum rising sharply below $1080 \mathrm{~cm}^{-1}$ is interpreted as the strongly broadened $Q$ line of the parallel band $\nu_{6}{ }^{*}$. Although quite uncertain because of the anomalous absorption pattern, the band center of the perpendicular band is estimated to lie between the lines 2 and 3 and the assignments of the rotational quantum numbers are given on this basis. An anomalous absorption pattern is indeed expected because of the strong coupling between the vibrations $\nu_{6}{ }^{*}$ and $\nu_{8}{ }^{*}$ through the rotation of the molecule. The zeta-value characterizing this coupling has been estimated, on the basis of the final potential constants, to be 0.54 , which is sufficiently large to produce the observed amount of distortion when the separation of band centers is small. Although this known interaction supports the qualitative correctness of the above choice of band centers, it is inconsistent with the latter in a quantitative sense (in that, in the absence of other couplings, an absorption head should appear at the unperturbed position of the $\perp$ band center. It may be noted that the interaction zeta referred to here gives a measure of the internal angular momentum associated with the superposition of two distinct normal vibrations and hence results in first-order energy contributions only when, as in the above case, such vibrations are accidentally degenerate.)

${ }^{8}$ G. Herzberg, Infrared and Raman Spectra of Polyatomic Molecules (D. Van Nostrand Company, Inc., New York, 1945).
The double peak in the envelope of the $\nu_{7}^{*}$ band is presumably occasioned by resonance interaction with the combination bands $\nu_{2+6}{ }^{*}$ and $\nu_{2+8}{ }^{*}$. The exact interpretation is obscure and the present measurements do not warrant a change in the value $\nu_{7}^{*}=2236 \mathrm{~cm}^{-1}$ reported by Stitt.

\section{INITIAL REDUCTION OF THE DATA}

The potential energy function for a molecule is generally represented as a power series in the relative atomic displacements, the coefficients associated with the quadratic terms constituting the potential constants which are the subject of this paper.

The normal frequencies, which correspond to atomic vibrations of infinitesimal amplitude, are functions of these potential constants. The fundamental frequencies, however, which are the quantities observed experimentally and which may differ by a few percent from the normal frequencies, depend principally upon the quadratic coefficients, but also to a lesser extent upon the so-called anharmonic terms which are cubic and quartic in the atomic displacements. When all of the simple combination and harmonic bands have been measured, the anharmonic contributions can be found and, through subtraction, the normal frequencies ascertained. It may be remarked that although the normal and fundamental frequencies may be in agreement within a few percent, quadratic potential constants determined by using fundamental rather than normal frequencies may often be in error by several times this amount. In the following discussion, fundamental and normal frequencies will be denoted by the symbols $\nu_{i}$ and $\omega_{i}$, respectively.

For ethane and heavy ethane, measurements of the very large number of simple combination bands are almost necessarily incomplete and one must rely on estimates of the anharmonicity corrections. A general procedure for the estimation of such corrections has been given by Dennison ${ }^{6}$ based on the Teller product rule and approximate relations between anharmonicity corrections of isotopic molecules. Thus, defining the anharmonicity factor, $x$, through the equation $\nu=(1-x) \omega$, the perturbation treatment of the higher order potential terms of isotopic dialomic molecules gives the correction ratio $x^{*} / x=\omega^{*} / \omega \cong \nu^{*} / \nu$. This same ratio is assumed to hold approximately for the similar vibrations of isotopic polyatomic molecules. Designating by $\mu_{i j}$ the mass coefficients appearing in the kinetic energy expression for one of the symmetry classes of vibrations, the Teller product rule gives a relation between the ratio of the determinants $\left|\mu_{i j}\right|$ and $\left|\mu_{i j}{ }^{*}\right|$ and the products of the normal frequencies, namely,

$$
\left|\mu_{i j}{ }^{*}\right| \frac{1}{2} /\left|\mu_{i j}\right|^{\frac{1}{1}}=\pi_{i}\left(\omega_{i} / \omega_{i}^{*}\right) \cong \pi_{i}\left(\nu_{i} / \nu_{i}^{*}-x_{i}\right) /\left(1-x_{i}\right) .
$$

As there are five symmetry classes for ethane, the product rule gives five additional relations among the anharmonicity factors. Many of the vibrations of ethane, such as the $\mathrm{C}-\mathrm{H}$ stretching and $\mathrm{H}-\mathrm{C}-\mathrm{H}$ 
angle bending vibrations are similar to those of methane, for which the anharmonicity factors are reasonably well known. For such vibrations, the anharmonicity factors of ethane may be chosen to agree with the known factors of methane subject, of course, to the equations given by the product rule. Thus, starting with the fundamental frequencies of ethane and heavy ethane, the anharmonicity corrections may be estimated and the set of normal frequencies obtained by means of the relations

$$
\begin{gathered}
x_{i}^{*} / x_{i}=\nu_{i}^{*} / \nu_{i} \\
\left|\mu_{i j}\right|^{\frac{1}{2}} /\left|\mu_{i j}{ }^{*}\right|^{\frac{1}{2}}=\pi_{i}\left(\nu_{i} / \nu_{i}^{*}-x_{i}\right) /\left(1-x_{i}\right) \\
x_{i} \text { (ethane) } \sim x_{i} \text { (methane) subject to Eq. (2). }
\end{gathered}
$$

It should be emphasized that these relations are only approximate and there has been no hesitation to make readjustments of the values from the ethane-methane correlation so as to conform to the empirical rules that anharmonicity factors are generally positive and are larger for the higher frequency vibrations.

Although the zeta-values are also slightly affected by the anharmonic potential terms, neither sufficient experimental data nor empirical procedure is available to make corrections. In the absence of resonance, these corrections are expected to be of the order $\Delta \zeta \sim\left(V_{1} / V_{0}\right)^{2}$ $\sim V_{2} / V_{0} \sim 0.01$, where $V_{0}, V_{1}$ and $V_{2}$ are typical quadratic, cubic, and quartic potential terms. The squared correction arises from and is proportional to the difference in zeta-values of the coupled vibrations, while the $V_{2}$ contribution arises from the interference between the anharmonic terms and the nondiagonal elements of the vibration-rotation interaction energy.

Table IV gives a listing of the fundamental frequencies and zeta-values of ethane and heavy ethane together with the estimated anharmonicity factors and normal frequencies.

The values for the fundamental frequencies and for the zetas have been taken directly from the summary of Raman data listed by Herzberg ${ }^{3}$ and from the work of Smith ${ }^{5}$ with the following exceptions where it appears that a reinterpretation of the experimental observations will yield more reliable numbers:

(1) Because of resonance interactions with combination vibrations, the fundamentals $\nu_{1}, \nu_{1}{ }^{*}, \nu_{5}, \nu_{5}{ }^{*}$, and $\nu_{10}$ appear in the spectrum as doublet bands. The "resonance free" values listed for these fundamentals are estimates furnished by the relative intensities of the doublet components and the relative doublet separations in ethane and heavy ethane. As an example of this process, consider the frequency $\nu_{5}$. The experimental spectrum shows two bands, one at 2895.60 and the other at $2954.05 \mathrm{~cm}^{-1}$, of which the former is of the order of twice the intensity of the latter. The existence of these two bands is undoubtedly due to the resonance interaction at $\nu_{5}$ and the overtone level $\nu_{8+11}$. The wave functions describing the resonanting levels are linear combinations of the wave functions for $\nu_{5}$ and $\nu_{8+11}$ and
Table IV. Fundamental frequencies, anharmonicity factors, normal frequencies, and zeta-values for ethane and heavy ethane.

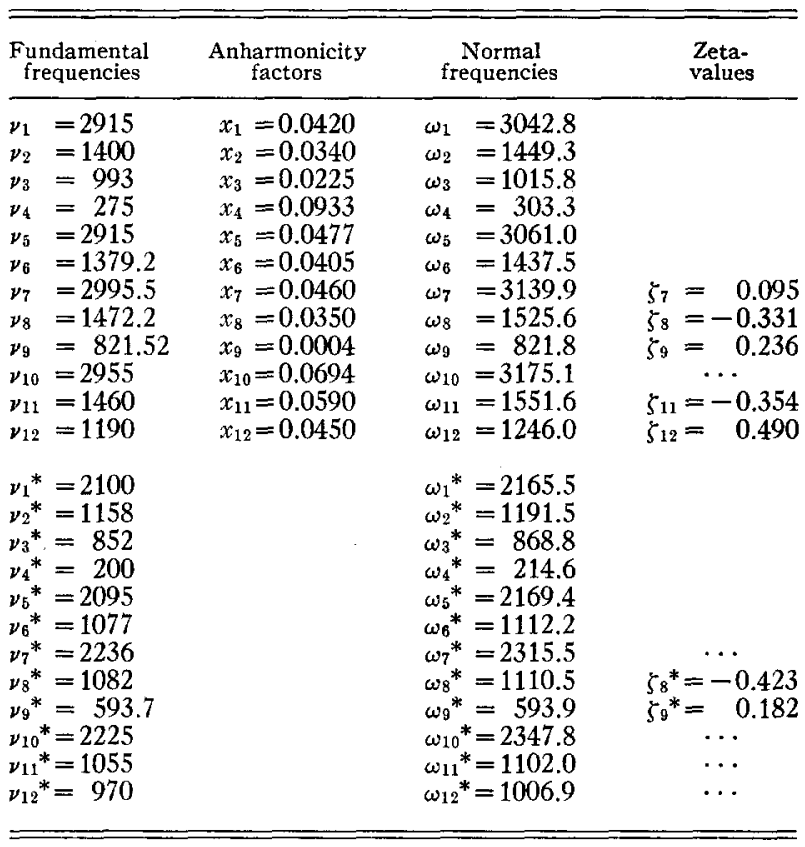

the square of the coefficients of the $\nu_{5}$ wave function will measure the intensity of the transition to that resonating level. It also measures the extent to which the energy of the resonating level depends upon the unperturbed energy level of $\nu_{5}$. Thus if the intensity ratio of the bands at 2895.6 and at 2954.0 is as $2: 1$, one may conclude that the unperturbed level

$$
\nu_{5}=[2(2895.6)+2954.0] / 3=2915 \mathrm{~cm}^{-1} .
$$

(2) The vibration $\nu_{2}$, called the "uncertain frequency" of ethane has not been observed directly and its frequency is estimated from the combination bands $\nu_{2+2}=2778 \mathrm{~cm}^{-1}$ and $\nu_{2+2}{ }^{*}=2300 \mathrm{~cm}^{-1} 10$ and the approximate isotope relations

$$
\nu_{2+2} \equiv 2 \nu_{2}\left(1-x^{\prime}\right) \rightarrow \nu_{2+2}{ }^{*} \cong 2 \nu_{2} *\left(1-x^{\prime} \nu_{2}{ }^{\prime} / \nu_{2}\right) .
$$

The resultant value $\nu_{2}=1400 \mathrm{~cm}^{-1}$ is in good agreement with values estimated from the combination bands $\nu_{2+6}=2753.3$ and $\nu_{2+9}=2218 \mathrm{~cm}^{-1}$. 9

(3) The value $\nu_{12}=1190 \mathrm{~cm}^{-1}$ is that obtained by Smith from the combination band $\nu_{1+12}$ and is consistent with estimates given by $\nu_{9+12}=2021.6 \mathrm{~cm}^{-110}$ and $\nu_{7+12}=4178.9 \mathrm{~cm}^{-1}$. These latter combination bands, which have the rotational spacings $7.60 \mathrm{~cm}^{-1}$ and 6.89

\footnotetext{
${ }^{9}$ It is the measurement of the $\nu_{2+9}$ by Smith which favors a value for $\nu_{2}$ higher than the value $\nu_{2}=1375 \mathrm{~cm}^{-1}$ originally given in reference 3 . It then becomes preferable to assign the $2778 \mathrm{~cm}^{-1}$ band as $\nu_{2+2}$ rather than $\nu_{6+6}$ as given also originally in reference 3 .

${ }^{10}$ As mentioned by Smith, the appearance in the infrared spectrum of a perpendicular band with large rotational spacing at $2021.6 \mathrm{~cm}^{-1}$ is the strongest argument in favor of the $D_{3 d}$ equilibrium configuration of ethane, since this position and rotational spacing forces the band's assignment as $\nu_{9+12}$, a combination band infrared active for the $D_{3 d}$ model but infrared inactive for the $\mathrm{D}_{3 h}$ model.
} 
$\mathrm{cm}^{-1}, 11$ respectively, determine the mean zeta-value $\zeta_{12}=0.490$.

(4) The value $\zeta_{12}=0.490$ given above is somewhat higher than the value 0.325 computed by Smith from his measured values of $\zeta_{10}$ and $\zeta_{11}$ together with the zeta-sum rule for the perpendicular inactive vibrations $\left(\zeta_{10}+\zeta_{11}+\zeta_{12}\right)=B / 2 A=0.128$. However, there is strong evidence that the vibration $\nu_{10}$ is involved in a resonance interaction with the combination band $\nu_{8+8}$ and hence that the associated zeta-value is anomalous. Thus, assuming such a coupling between $\nu_{10}$ and $\nu_{8+8}$ which gives rise to the complex vibrations $\left[\nu_{10}\right]_{a}$ and $\left[\nu_{10}\right]_{b}$, then an associated zeta-value, say $\left[\zeta_{10}\right]_{a}$ will be an average of the unperturbed values $\zeta_{10}=-0.008$ (as obtained by zeta-sum rule and tabulated values of $\zeta_{11}$ and $\left.\zeta_{12}\right)$ and $\zeta_{8+8}=-2 \zeta_{8}=0.662 .{ }^{12}$ weighted according to the composition of $\left[\nu_{10}\right]_{a}$ in terms of $\nu_{10}$ and $\nu_{8+8}$. In the different combination vibrations $\left[\nu_{i+10}\right]$, the computed $\left[\zeta_{10}\right]$ values are expected to be different due to

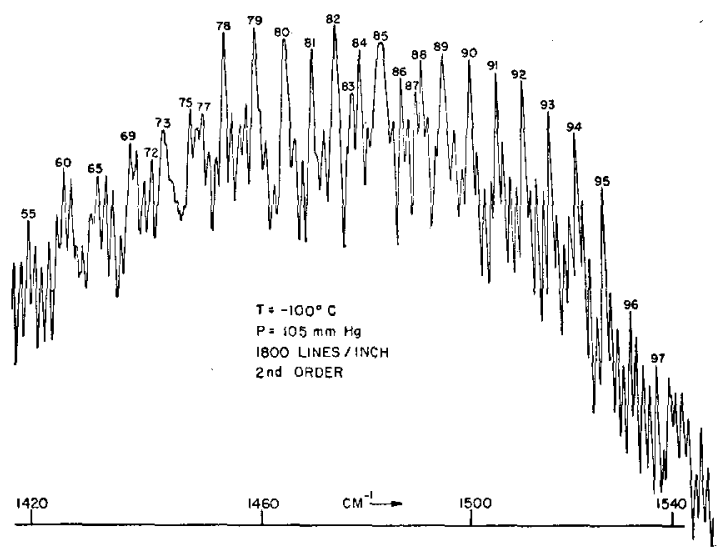

Fig. 5. Absorption by the $\nu_{8}$ band of ethane (after L. G. Smith, reference 5). The nature of the irregularities in the fine structure of this band furnishes an independent check on the $D_{3 d}$ equilibrium configuration of ethane.

the variation in completeness of resonance. These consequences are amply shown by Smith's measurements on the following combination bands.

$$
\begin{aligned}
& {\left[\nu_{1+10}\right]=3258 \mathrm{~cm}^{-1} \quad\left[\nu_{10}\right]=2968 \mathrm{~cm}^{-1}} \\
& {\left[\zeta_{4+10}\right]=0.164 \rightarrow\left[\zeta_{10}\right]=0.164} \\
& {\left[\nu_{6+10}\right]=4334 \mathrm{~cm}^{-1} \rightarrow\left[\nu_{10}\right]=2955 \mathrm{~cm}^{-1}} \\
& {\left[\zeta_{6+10}\right]=0.147 \rightarrow\left[\mathrm{cm}^{-1} \rightarrow\left[\nu_{10}\right]=0.147 .\right.} \\
& {\left[\nu_{8+10}\right]=4416 \mathrm{~cm}^{-1}} \\
& {\left[\zeta_{8+10}\right]=-0.039 \rightarrow\left[\zeta_{10}\right]=0.370} \\
& {\left[\nu_{7+10}\right]=5950 \mathrm{~cm}^{-1} \rightarrow\left[\nu_{10}\right]=2955 \mathrm{~cm}^{-1}} \\
& {\left[\zeta_{7+10}\right]=-0.228 \rightarrow\left[\zeta_{10}\right]=0.135 .}
\end{aligned}
$$

${ }^{11}$ Smith reported these spacings as 7.64 and $7.02 \mathrm{~cm}^{-1}$ respectively, but reexamination of his data seems to indicate the above listed values to be preferable.

${ }_{12}$ M. Johnston and D. M. Dennison, Phys. Rev. 48, 868 (1935) show that the dipole coubling between a combination level and
Further support for the $\nu_{10}, \nu_{8+8}$ interaction is offered by the Raman data of Lewis and Houston ${ }^{13}$ if the Stokes lines in the vicinity of $3000 \mathrm{~cm}^{-1}$ and the single line at $2939 \mathrm{~cm}^{-1}$ are interpreted respectively as the ${ }^{S} Q^{K}$ lines and the first ${ }^{\circ} Q^{K}$ line of the band $\left[\nu_{10}\right]$. With this interpretation, one obtains $\left[\nu_{10}\right]=2947 \mathrm{~cm}^{-1}$ and a rotational spacing of $10.0 \mathrm{~cm}^{-1}$ which corresponds to the zeta-value $\left[\zeta_{10}\right]=0.444$.

(5) As the torsional vibration is both Raman and infrared inactive, the spectroscopic determination of its fundamental frequency can be obtained only from combination bands. For $\mathrm{C}_{2} \mathrm{H}_{6}$, Smith has determined the average value $\nu_{4}=290 \mathrm{~cm}^{-1}$ from the two bands $\nu_{4+11}$ and $\nu_{4+10}$ while for $\mathrm{C}_{2} \mathrm{D}_{6}$, Stitt's measurement $\nu_{4+10} *$ $=2415 \mathrm{~cm}^{-1}$ gives $\nu_{4} *=190 \mathrm{~cm}^{-1}$. For these two values, the ratio $\nu_{4} / \nu_{4}^{*}=1.526$ differs so markedly from the product rule ratio, $\left(m^{*} / m\right)^{\frac{1}{2}}$, as to indicate a real inconsistency. Although, in general, spectroscopic methods are the more accurate, in this case specific heat measurements give the more concordant results of $\nu_{4}=275 \mathrm{~cm}^{-1}$ and $\nu_{4}{ }^{*}=200 \mathrm{~cm}^{-1},{ }^{14}$ and it is these latter values which are listed in Table IV.

The perturbation of the $\nu_{10}$ band prevents the use of the observed rotational spacings of the perpendicular inactive bands for a check on the small moment of inertia of ethane as determined by Smith from the rotational spacings $\Delta \nu_{7}, \Delta \nu_{8}$, and $\Delta \nu_{9}$. Although the $\nu_{8}$ band is also perturbed, as may be seen from the spectrogram given in Fig. 5 and reproduced from Smith's paper, this perturbation is small and the resolution of the fine structure is sufficient to permit simple interpretation and consequent determination of the normal or unperturbed rotational spacing. The general results of an analysis of the $\nu_{8}$ band have been cited by Smith but, as the perturbation scheme involved requires the $D_{3 d}$ equilibrium configuration for ethane (and hence constitutes a check on the correctness of this model), it seems worthwhile to include here a summary of the method as well as the numerical agreement between the observed and predicted positions of the main absorption lines. The procedure consists of two parts. First, the assignment of a quantum number, $K$, to each $Q$ line, and second, the determination of perturbation parameters required to match the observed and predicted line positions.

For the assignment of quantum numbers, one takes advantage of the feature that the fine structure of $\nu_{8}$ becomes regular at the high frequency side of the band indicating that the perturbing forces tend to vanish in this direction. Using the $Q$ line spacing in this high frequency region to establish approximate values for the small moment of inertia and the zeta-value for this

the vibrational ground state is such that if $l_{1}, l_{2}, \ldots$ represent the internal angular momenta quantum numbers, the apparent zetavalue for the combination band is $\zeta=+\left(\Sigma l_{i} \zeta_{i}\right)$ or $-\left(\Sigma l_{i} \zeta_{i}\right)$ as $\left|\Sigma l_{i}\right|=3 n+1$ or $3 n-1$, where $n$ is any integer.

${ }^{13}$ C. M. Lewis and W. V. Houston, Phys. Rev. 44, 903 (1933).

${ }^{14}$ Kistiakowsky, Lacher, and Stitt, J. Chem. Phys. 7, 289 (1939). 
band, a theoretical intensity distribution for the equivalent unperturbed band may be calculated. This predicted intensity distribution is pictured in Fig. 6. An unusual situation occurs in the $\nu_{8}$ band in that, due to a coincidence, the spacing of the lines in the sub-bands for which $\Delta J= \pm 1$ are almost exactly $\frac{1}{4}$ of the $Q$ line spacing. This means that the sub-band lines will bunch. together into groups of three equispaced lines lying between the individual $Q$ lines. These groups of lines have characteristic intensity patterns. Thus, for example, between the $K$ lines 5 and 6 , the low frequency member of the triplet is the most intense due to the large contribution of the first $R$ line of the $K=4$ subband. Similarly between 6 and 7 the middle member is intense, and between 7 and 8 the last member is intense, this being due, respectively, to the first $R$ lines of the $K=5$ and $K=6$ sub-bands. These features are exactly matched by the intensities of the substructure lines found by Smith between the $Q$ lines which he designated as 91 and 92, 92 and 93, and 93 and 94, respectively. It will be noted that between the $K$ lines 10 and 11 , the substructure lines are predicted to be as intense as the $R$ lines and that this condition is found to obtain in the corresponding position of the observed band, namely between lines 96 and 97 .

From his examination of the $\nu_{8}$ band, Smith concluded that two distinct regions of perturbation were present and suggested that these perturbations were the result of Fermi and Coriolis interactions with the combination band $\nu_{4+12}$ which presumably lies in this region. The stepwise assignments of the $Q$ lines starting from the high frequency side do indeed give two resonance like curves for the function $\nu_{8}(K)$. However, all the observed $Q$ lines are not accounted for by this procedure and these "extra" $Q$ lines do not fall on the resonance curves. Yet, the existence and positions of these "extra" lines are, as will be shown, actually a consequence of the interaction scheme suggested by Smith. From the line assignments given in Table V, the unperturbed rotational spacing and band center can be determined without any perturbation calculation. For these assignments indicate that the resonances occur near the middle of the band and hence the line positions at the high and low frequency sides are approaching asymptotically the hypothetical positions of the resonance free lines. A measurement of these two asymptotes permits the evaluation of the normal band center, the rotational spacing, and the convergence of the resonance free lines. This procedure gives $\nu_{8}=1472.2$ $\mathrm{cm}^{-1}$ and $\Delta \nu_{8}=5.563 \mathrm{~cm}^{-1}$ (at the band center).

For the quantitative determination of the structure of $\nu_{8}$, one has three essential perturbation parameters, (1) the strength, $\Delta$, of the Fermi coupling term (arising from an anharmonic potential term such as $\xi_{4} \rho_{12} \rho_{8} \sin \left(\phi_{8}-\phi_{12}\right)$ where $\xi_{4}$ is the torsional normal coordinate, and $\rho_{8}, \phi_{8}$ and $\rho_{12}, \phi_{12}$ are the polar normal coordinates associated with the vibrations $\omega_{8}$ and $\omega_{12}$ ),

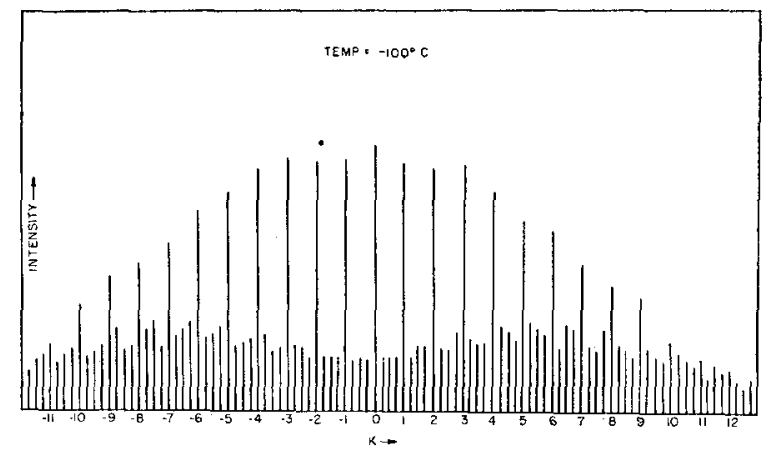

FIG. 6. Theoretical intensity distribution for the unperturbed $\nu_{8}$ band. The quantum number $K$ refers to that of the ground vibrational state.

(2) the strength, $f$, of the Coriolis coupling term (arising from the vibration-rotation interaction $\left(P_{x} p_{x}+P_{y} p_{y}\right)$ / $I_{x}$. Although this interaction strength depends on the rotational quantum numbers as $C[(J-K)(J+K+1)]^{3}$, one may use a value averaged over $J$ which near the band center is essentially $C \vec{J}=f$, a number independent of $K$. In the harmonic oscillator approximation there is no Coriolis coupling between $\nu_{8}$ and $\nu_{4+12}$ and the nonvanishing of the coupling coefficient $C$ depends on the existence of appropriate anharmonic potential terms), and (3) the separation, $a$, between the unperturbed band centers of $\nu_{8}$ and $\nu_{4+12}$. The energy contributions due to the Fermi and Coriolis interactions are determined from the eigenvalues of the $4 \times 4$ matrices (see Herzberg for the selection rules governing such

TABLE V. Line assignments for the $\nu_{8}$ band. ${ }^{\mathrm{a}}$

\begin{tabular}{crrrr}
\hline Line no. & $K^{\prime \prime}$ & $K^{\prime}$ & \multicolumn{1}{c}{$\mathrm{cm}^{-1}$} & Perturbation $\left(\mathrm{cm}^{-1}\right)$ \\
\hline 55 & 10 & 9 & 1418.71 & -0.39 \\
60 & 9 & 8 & 1424.56 & -0.33 \\
65 & 8 & 7 & 1430.34 & -0.31 \\
69 & 7 & 6 & 14335.65 & -0.74 \\
72 & 6 & 5 & 1439.63 & -2.47 \\
73 & 6 & 5 & 1441.36 & -0.74 \\
75 & 5 & 4 & 1446.38 & -1.41 \\
77 & 5 & 4 & 1448.52 & 0.73 \\
78 & 4 & 3 & 1452.41 & -1.04 \\
79 & 3 & 2 & 1457.91 & -1.18 \\
80 & 2 & 1 & 1463.48 & -1.21 \\
81 & 1 & 0 & 1468.45 & -1.84 \\
82 & 0 & 1 & 1472.95 & -2.90 \\
83 & 0 & 1 & 1475.87 & 0.02 \\
84 & 1 & 2 & 1477.78 & -3.91 \\
& 1 & 2 & & 0.14 \\
85 & & & 1481.49 & -5.41 \\
& 2 & 3 & & 4.25 \\
86 & 1 & 2 & 1485.64 & 1.38 \\
87 & 2 & 3 & 1488.28 & 2.39 \\
88 & 2 & 3 & 1489.29 & 1.29 \\
89 & 3 & 4 & 1493.68 & 1.05 \\
90 & 4 & 5 & 1498.90 & 0.84 \\
91 & 5 & 6 & 1504.13 & 0.77 \\
92 & 6 & 7 & 1509.47 & 0.56 \\
93 & 7 & 8 & 1514.65 & 0.40 \\
94 & 8 & 9 & 1519.85 & 0.41 \\
95 & 9 & 10 & 1525.14 & 0.41 \\
96 & 10 & 11 & 1530.51 & \\
97 & 11 & 12 & 1535.80 & \\
\hline \hline
\end{tabular}

see reference 5 , 


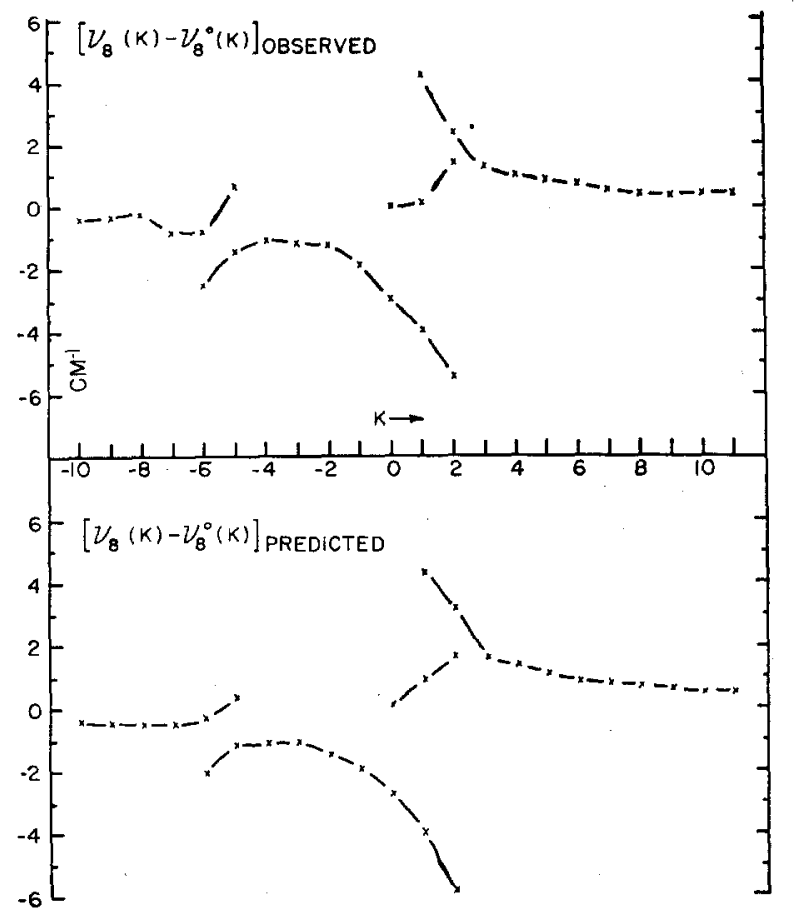

FIG. 7. Observed and calculated perturbation of the $Q$ lines of $\nu_{8}$.

interactions)

$$
\begin{array}{cccc}
E_{8}+(K) & \Delta & 0 & f \\
-\Delta & E_{4+12^{+}}(K) & -f & 0 \\
0 & f & E_{8}-(K+1) & -\Delta \\
-f & 0 & \Delta & E_{4+12}^{-}(K+1)
\end{array}
$$

where $E_{8}{ }^{+}(K)$ is the energy of the $K$ th rotational level of the state $\nu_{8}{ }^{+}$in the absence of the perturbing terms $\Delta$ and $f$. Thus

$$
E_{8}{ }^{+}(K)=(A-B) K^{2}-2 A \zeta_{8} K
$$

Using Smith's initial values for the moments of inertia and zeta-values, ${ }^{15}$ one obtains the perturbation function plotted in Fig. 7 with the choice

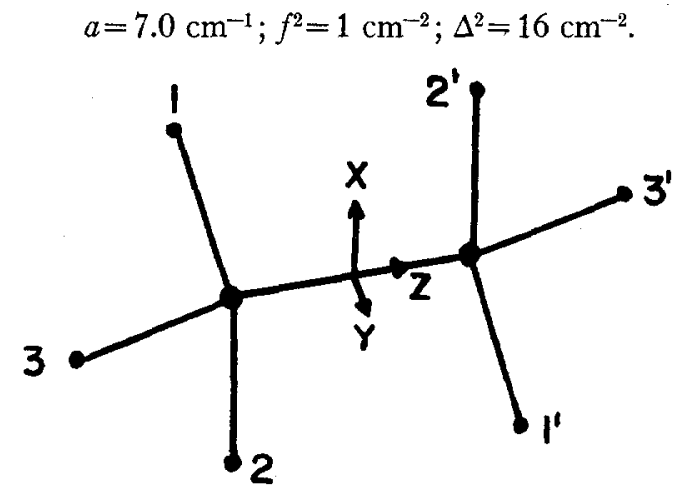

FIG. 8. Schematic diagram of the ethane molecule and the adopted indexing of the hydrogen and carbon atoms.

${ }^{15}$ L. G. Smith and W. N. Woodward, Phys. Rev. 61, 386(A) (1942).
Although the agreement between the predicted and observed perturbation functions might be improved somewhat by a more careful determination of the parameters $a, f$, and $\Delta$, the above choice is sufficiently close to show the essential correctness of the perturbation scheme. The "extra" $Q$ lines which are one of the characteristic features of the spectrum arise from the coupling, by way of $\nu_{4+12}$, of two different levels of $\nu_{8}$ which normally make transitions to two different ground vibrational states (as contrasted, say, to the coupling of two levels of a perpendicular band via rotational interaction with a common third level of a parallel band as in $\nu_{6}{ }^{*}$ and $\nu_{8}{ }^{*}$ where no "extra" lines are expected).

\section{DETERMINATION OF THE GENERAL POTENTIAL CONSTANTS}

The twenty-two independent potential constants compatible with the symmetry of ethane are defined by means of the set of symmetry coordinates pictured in Fig. 9 and are evaluated in groups-one group for each symmetry class of vibrations. It will be noted that these coordinates uniquely specify the configuration of the molecule whatever the ratio of carbon and hydrogen masses may be. They are thus geometric coordinates in the sense used by Johnston and Dennison ${ }^{12}$ (thus, in terms of these coordinates, the potential energy expression is the same for both ethane and heavy ethane). The appropriate expressions for the kinetic and potential energies of each class of vibrations are given in the following order: (1) $E_{u}$; (2) $E_{g}$; (3) $A_{1 g}$; (4) $A_{2 u}$; and (5) $A_{1 u}$. For the degenerate classes, the $z$ components of internal angular momenta are also given.

In the following, $m, m^{*}$, and $M$ will designate, respectively, the masses of the hydrogen, deuterium, and carbon atoms.

\section{A. Perpendicular Active Vibrations $\left(E_{u}\right)$}

The coordinates $\xi_{1}, \eta_{1}$ represent the well-known degenerate vibrations of a triatomic molecule with equal atoms set at the corners of an equilateral triangle. A displacement of the coordinate $\xi_{1}$ is such that the atoms of one of the hydrogen triangles execute the displacements shown in diagram (1a) of Fig. 9, while the atoms of the other triangle are displaced as in (1b). The actual displacement of each of the six atoms may be shown to be equal and is chosen to be $\left[1 /(6)^{\frac{1}{2}}\right] \xi_{1}$. The $\eta_{1}$ displacements are the usual complements of $\xi_{1}$ and will not be shown. In both $\xi_{1}$ and $\eta_{1}$ the carbon atoms are not displaced and hence the kinetic energy is just $\frac{1}{2} m\left(\dot{\xi}_{1}{ }^{2}+\dot{\eta}_{1}{ }^{2}\right)$ for light ethane and $\frac{1}{2} m^{*}\left(\dot{\xi}_{1}{ }^{2}+\dot{\eta}_{1}{ }^{2}\right)$ for heavy ethane.

The displacements $\xi_{2}$ and $\eta_{2}$ are again ones in which neither the carbon atoms nor the center of gravity of each $\mathrm{H}_{3}$ group move and consist in a rocking of each of the hydrogen triangles through an angle $\alpha$. If now $\xi_{2}=S \alpha$ where $S$ is the equilibrium side of a hydrogen triangle, the kinetic energy associated with this motion is $\frac{1}{2} m\left(\xi_{2}{ }^{2}+\dot{\eta}_{2}{ }^{2}\right)$ or $\frac{1}{2} m^{*}\left(\xi_{2}{ }^{2}+\dot{\eta}_{2}{ }^{2}\right) . \eta_{2}$ is of course 


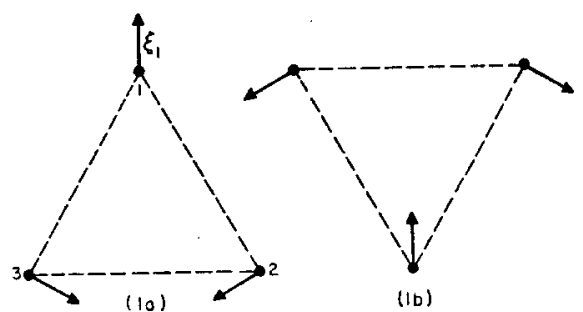

(1) Distortion of the
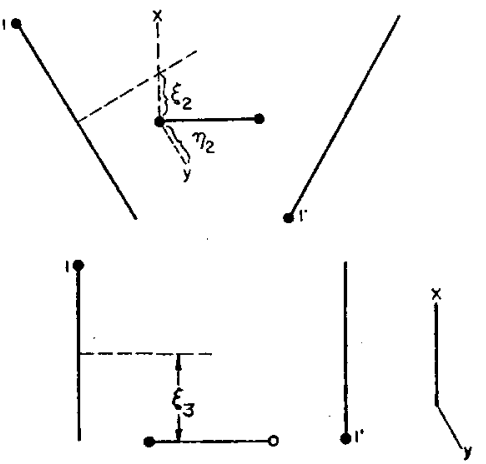

(a)
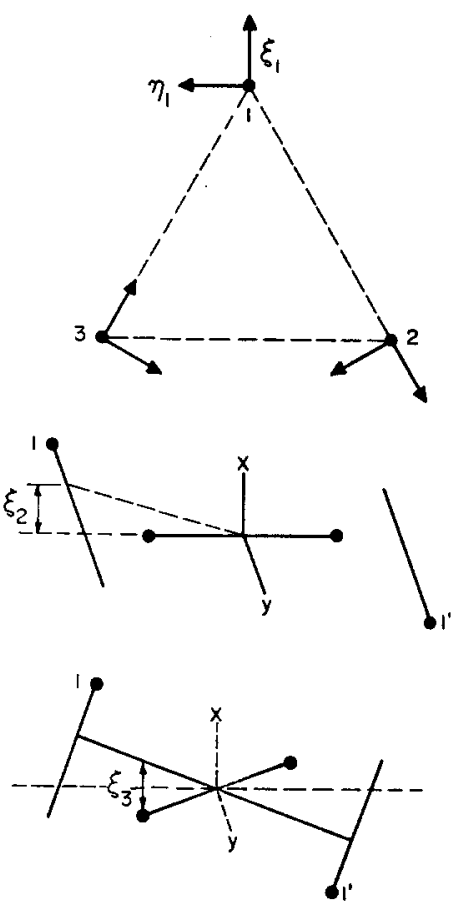
(3) Rocking at the
"hydrogen cylinder"

(3) Translation of the "hydrogen triangles" (1) Distortion of the
"hydrogen triangles" (2) Shearing of the
"hydrogen triangles"

(b)

Fig. 9. Definition of the set of symmetry coordinates used for the representation of the potential energy function of ethane. (a) Coordinates for expressing the perpendicular active vibrations. (b) Coordinates for expressing the perpendicular inactive vibrations. (c) Coordinates for expressing the parallel inactive vibrations. (d) Coordinates for expressing the parallel active vibrations. (e) Coordinate for expressing the torsional vibration.
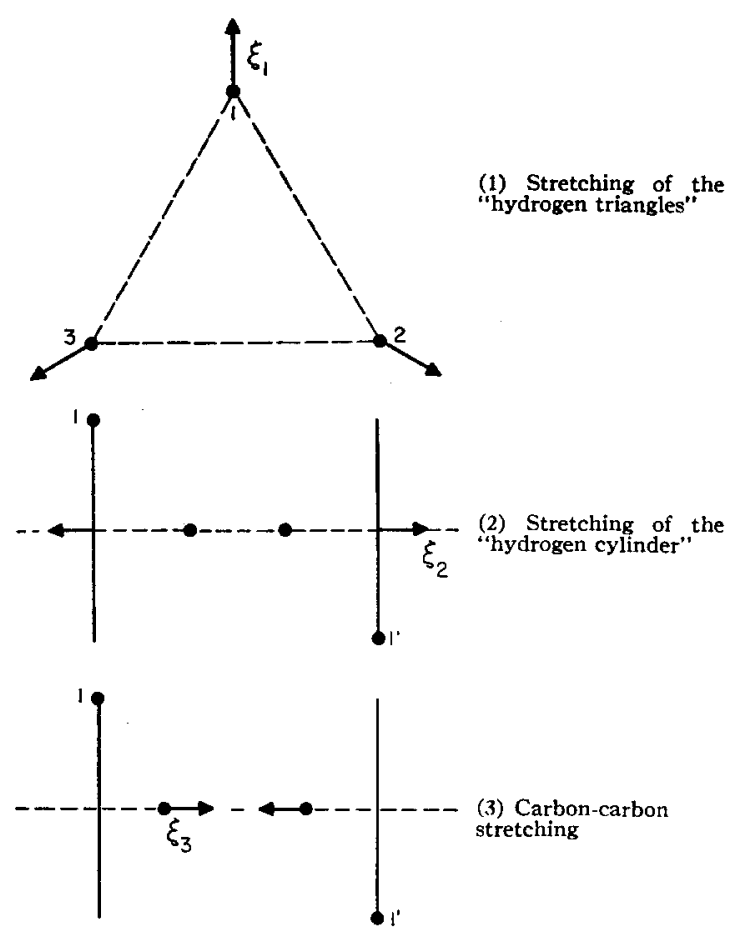

(c)

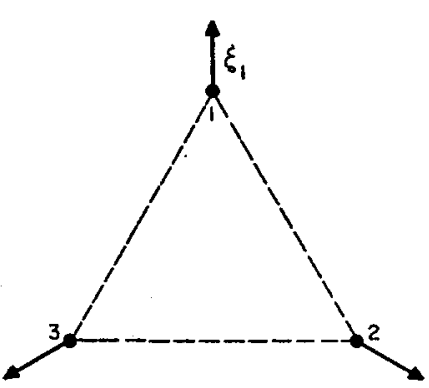

(1) Stretching of the "hydrogen triangles"

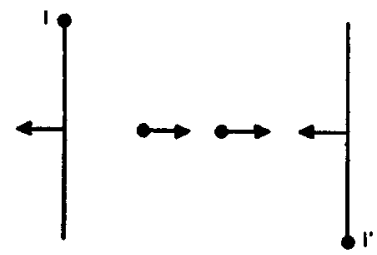

(d)

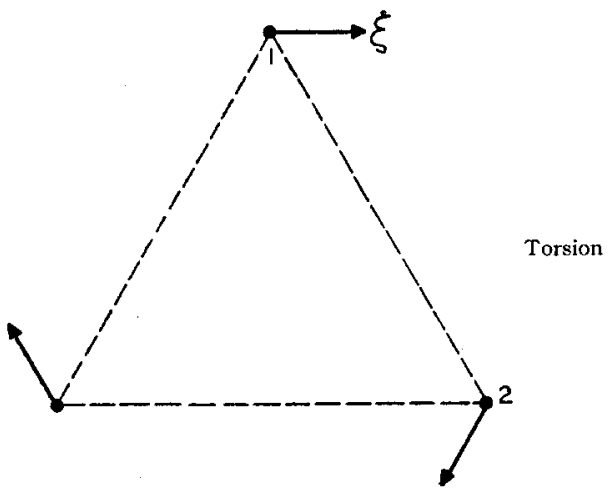

(e) 
TABLE VI. Potential constants of ethane based on coordinate system defined in Fig. 9. All constants are expressed in units of $10^{6}$ dynes $/ \mathrm{cm}$.

(a) Potential constants assaciated with the perpendicular active vibrations.

$\begin{array}{ll}a_{11}=2.992 & a_{12}=1.182 \\ a_{22}=1.436 & a_{13}=1.790 \\ a_{33}=3.203 & a_{23}=1.693\end{array}$

(b) Potential constants associated with the perpendicular inactive vibrations.

$\begin{array}{ll}b_{11}=3.244 & b_{12}=1.778 \\ b_{22}=3.159 & b_{13}=1.401 \\ b_{33}=1.935 & b_{23}=1.456\end{array}$

(c) Potential constants associated with the parallel inactive vibrations.

$\begin{array}{ll}c_{11}=5.330 & c_{12}=0.705 \\ c_{22}=1.238 & c_{13}=0.431 \\ c_{33}=0.789 & c_{23}=0.331\end{array}$

(d) Potential constants associated with the parallel active vibrations.

$d_{11}=5.484 \quad d_{12}=0.578$ $d_{22}=1.305$

(e) Torsion constant: $e=0.0546$

similar to $\xi_{2}$ but the axis of the rocking motion is turned through $90^{\circ}$.

The displacements $\xi_{3}$ and $\eta_{3}$ are ones where the hydrogen triangles are both displaced in one direction without rocking and the carbons are displaced in the reverse direction. For convenience, the displacement of the carbon atoms relative to the $\mathrm{H}$-triangles has been chosen to be $(3 m+M / 6 M)^{\frac{1}{2}}$ times $\xi_{3}$ or $\eta_{3}$ and hence the kinetic energy for light ethane is $\frac{1}{2} m\left(\dot{\xi}_{3}{ }^{2}+\dot{\eta}_{3}{ }^{2}\right)$ and for heavy ethane is $\frac{1}{2} m^{*}\left(3 m+M / 3 m^{*}+M\right)\left(\dot{\xi}_{3}{ }^{2}+\dot{\eta}_{3}{ }^{2}\right)$.

In each of these displacements it will be noted that there is neither a net rotation nor change in center of gravity of either the light or heavy ethane molecule. (These requisite features also obtain for the individual displacements defined for the other symmetry classes.) The choice of the coordinates has been such that there exist no cross product terms in the kinetic energy. Collecting the above results we have

$$
\begin{aligned}
T & =\frac{1}{2} m\left(\dot{\xi}_{1}{ }^{2}+\dot{\xi}_{2}{ }^{2}+\dot{\xi}_{3}{ }^{2}+\dot{\eta}_{1}{ }^{2}+\dot{\eta}_{2}{ }^{2}+\dot{\eta}_{3}{ }^{2}\right) \\
T^{*} & =\frac{1}{2} m^{*}\left(\dot{\xi}_{1}{ }^{2}+\dot{\xi}_{2}{ }^{2}+\dot{\xi}_{3}{ }^{2} / \sigma+\dot{\eta}_{1}{ }^{2}+\dot{\eta}_{2}{ }^{2}+\dot{\eta}_{3}{ }^{2} / \sigma\right) ; \\
\sigma & =\left(3 m^{*}+M\right) /(3 m+M) \\
V & =V^{*}=\frac{1}{2} \Sigma a_{i j}\left(\xi_{i} \xi_{j}+\eta_{i} \eta_{j}\right) \\
p_{z} & =p_{z}{ }^{*}=-1\left(\xi_{1} p_{\eta_{1}}-\eta_{1} p \xi_{1}\right)+1\left(\xi_{3} p_{\eta_{3}}-\eta_{3} p \xi_{3}\right)
\end{aligned}
$$

where $p_{\eta_{1}}$ is the momentum conjugate to $\eta_{1}$, etc.

For the estimation of the normal frequencies we have adopted the following set of anharmonicity factors: $X_{7}=0.0460, X_{8}=0.0350, X_{9}=0.0004$. The values of $X_{7}$ and $X_{8}$ were chosen, in accordance with the principles outlined in Sec. III, to agree roughly with the anharmonicity factors 0.046 and 0.039 of the corresponding triply degenerate frequencies of methane. ${ }^{7}$ The value of $X_{8}$ was arbitrarily lowered to 0.0350 so that the Teller product rule would give a positive value for $X_{9}$. By using least squares and a weighting scheme based on the estimate that a 1 percent error in a normal frequency is equivalent to an error of 0.02 in a zetavalue, the six potential constants, $a_{i j}$, are readily calculated from the determinant giving the normal frequencies and from the theoretical expressions for the zetas. They are listed in Table VI and yield the following calculated normal frequencies and zeta-values which may be compared with the observed quantities.

\begin{tabular}{cccrc} 
& \multicolumn{2}{c}{ Normal frequencies $\left(\mathrm{cm}^{-1}\right)$} & \multicolumn{2}{c}{ Zeta-values } \\
Vibration & Predicted & Observed & Predicted & Observed \\
$\nu_{7}$ & 3136.9 & 3139.9 & 0.098 & 0.095 \\
$\nu_{8}$ & 1526.9 & 1525.6 & -0.334 & -0.331 \\
$\nu_{9}$ & 825.1 & 821.8 & 0.236 & 0.236 \\
$\nu_{7}{ }^{*}$ & 2321.3 & 2315.5 & 0.213 & $\ldots$ \\
$\nu_{8}{ }^{*}$ & 1109.3 & 1110.5 & -0.403 & -0.423 \\
$\nu_{8}{ }^{*}$ & 595.2 & 595.3 & 0.190 & 0.182
\end{tabular}

The unitary matrices which connect the normal vibrations with the displacements $\xi_{1}, \xi_{2}, \xi_{3}$ (or $\eta_{1}, \eta_{2}, \eta_{3}$ ) have been calculated to have the following numerical elements.

$$
\begin{aligned}
& \begin{array}{llll}
S_{71}=0.601 & S_{72}=0.422 & S_{73}=0.678 \\
S_{81}=-0.797 & S_{82}=0.253 & S_{83}=0.548 \\
S_{91}=0.059 & S_{92}=-0.871 & S_{93}=0.489
\end{array}\left(\begin{array}{l}
\xi_{7} \\
\xi_{8} \\
\xi_{9}
\end{array}\right)=S\left(\begin{array}{l}
\xi_{1} \\
\xi_{2} \\
\xi_{3}
\end{array}\right)
\end{aligned}
$$

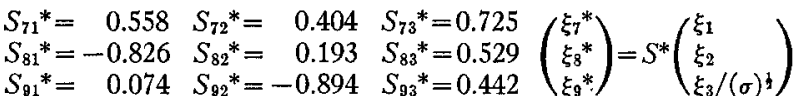

These elements are of value in determining the actual motions of the atoms. For example, the normal vibration $\omega_{7}$ consists of 0.601 of the displacement $\xi_{1}$ on which has been superposed 0.422 of displacement $\xi_{2}$ and 0.678 of $\xi_{3}$. The zeta-value associated with $\omega_{7}$ is found by weighting the zeta-values of $\xi_{1}, \xi_{2}$, and $\xi_{3}$, namely $-1,0,+1$, respectively, with the squares of the matrix elements. Thus

$$
\zeta_{7}=(-1)(0.601)^{2}+(0)(0.422)^{2}+(1)(0.678)^{2}=0.098 .
$$

\section{B. Perpendicular Inactive Vibrations $\left(E_{q}\right)$}

The coordinates $\xi_{1}, \eta_{1}$ are the same as the identically labelled coordinates of the $E_{u}$ class except for a reversal of phase in the atomic displacements in the opposing hydrogen triangle [(1b) of Fig. 9].

In the displacement $\xi_{2}$, there is a rotation of each hydrogen triangle about its center together with a counter rotation of the triangle centers about the molecule center of such relative magnitude that no change in angular momentum occurs. For convenience, the scale of $\xi_{2}$ is chosen such that the displacement of a triangle center is $\left(I_{Z} / 12 I_{c}\right)^{\frac{1}{2}}$ times $\xi_{2}$ or $\eta_{2}$, where $I_{Z}$ is the large moment of inertia of ethane and $I_{c}$ is the large moment of the hydrogen "cylinder," that is, the structure consisting solely of the six hydrogen atoms in their equilibrium positions. The kinetic energy contributions for light and heavy ethane are then $\frac{1}{2} m\left(\dot{\xi}_{2}{ }^{2}+\dot{\eta}_{2}{ }^{2}\right)$ and $\frac{1}{2} m^{*}\left(\dot{\xi}_{2}{ }^{2}+\dot{\eta}_{2}{ }^{2}\right)$. 
In the displacement $\xi_{3}$, the hydrogen cylinder rocks through an angle $\alpha_{1}$ while the carbon atoms counter rotate about the molecule center through an angle $\alpha_{2}$ such that there is no change in angular momentum. Denoting the distance of a carbon atom from the molecule center by $S, \xi_{3}$ is chosen such that $S\left(\alpha_{1}+\alpha_{2}\right)$ $=\left(m I_{x} / 2 M I_{c}\right)^{\frac{1}{3}} \xi_{3}$ and hence the kinetic energy for light ethane is $\frac{1}{2} m\left(\dot{\xi}_{3}{ }^{2}+\dot{\eta}_{3}{ }^{2}\right)$ and for heavy ethane is $\frac{1}{2} m^{*}\left(I_{x} / I_{x}{ }^{*}\right)\left(\dot{\xi}_{3}{ }^{2}+\dot{\eta}_{3}{ }^{2}\right)$. Again the choice of coordinates has been such that there exist no cross product terms in the kinetic energy and one obtains the following expressions.

$$
\begin{aligned}
T= & \frac{1}{2} m\left(\dot{\xi}_{1}{ }^{2}+\dot{\xi}_{2}{ }^{2}+\dot{\xi}_{3}{ }^{2}+\dot{\eta}_{1}{ }^{2}+\dot{\eta}_{2}{ }^{2}+\dot{\eta}_{3}{ }^{2}\right) \\
T^{*}= & \frac{1}{2} m^{*}\left(\dot{\xi}_{1}{ }^{2}+\dot{\xi}_{2}{ }^{2}+\dot{\xi}_{3}{ }^{2} / \sigma+\dot{\eta}_{1}{ }^{2}+\dot{\eta}_{2}{ }^{2}+\dot{\eta}_{3}{ }^{2} / \sigma\right) \\
\sigma= & I_{x}{ }^{*} / I_{x} \\
V= & V^{*}=\frac{1}{2} \Sigma b_{i j}\left(\xi_{i} \xi_{j}+\eta_{i} \eta_{j}\right) \\
p_{z}= & -1\left(\xi_{1} p_{\eta_{1}}-\eta_{1} \xi_{1}\right)+\left(I_{Z} / 2 I_{c}\right)\left(\xi_{2} p_{\eta_{2}}-\eta_{2} p \xi_{2}\right) \\
& +\left\{1-\left(I_{Z} / 2 I_{c}\right)+\left(I_{Z} / 2 I_{x}\right)\right\}\left(\xi_{3} p_{\eta_{3}}-\eta_{3} p \xi_{3}\right) \\
& +\left\{\left(I_{z} / 2 I_{c}\right)\left[1-\left(I_{z} / 2 I_{c}\right)\right]\left[1-\left(I_{c} / I_{x}\right)\right]\right\}^{\frac{1}{2}} \\
& \times\left\{\left(\xi_{2} p_{\eta_{3}}-\eta_{3} p \xi_{2}\right)+\left(\xi_{3} p_{\eta_{2}}-\eta_{2} \xi_{3}\right)\right\} .
\end{aligned}
$$

With the coordinates $\xi_{1}{ }^{*}=\xi_{1} ; \xi_{2}{ }^{*}=\xi_{2} ; \xi_{3}{ }^{*}=\xi_{3} /(\sigma)^{\frac{1}{2}}$, the expression for the vibrational angular momentum of $\mathrm{C}_{2} \mathrm{D}_{6}$ is the same as $p_{z}$ above but with all quantities starred. ${ }^{16}$ The symbol $I_{c}$ appearing in $p_{z}$ refers as before to that part of the large moment of inertia of ethane contributed by the six hydrogen atoms. The values for the six potential constants $b_{i j}$ were determined from the five independent normal frequencies and the value of $\zeta_{11}$. The experimental value for $\zeta_{12}$, being obtained from combination bands, was not considered sufficiently accurate to be weighted in the determination of the potential constants. The values of the $b_{i j}$ listed in Table VI thus predict back the normal frequencies and the $\zeta_{11}$ value exactly and yield the value $\zeta_{12}=0.413$ as compared with the observed value of 0.490 . The as yet unobserved $\zeta$ values of heavy ethane are predicted to be $\zeta_{10}{ }^{*}=0.190, \zeta_{11}{ }^{*}=-0.395$, and $\zeta_{12}{ }^{*}=0.383$.

The unitary matrices connecting the normal vibrations with the displacements $\xi_{1}, \xi_{2}, \xi_{3}$ have the following numerical elements.

\footnotetext{
${ }^{16}$ The choice of coordinates such that the kinetic energy expression for ethane has a diagonal form and equal coefficients gives rise to two simplifying features: first, the transformation matrix $S_{i \alpha}$, connecting the symmetry coordinates $\xi_{i}$ with the normal coordinates $\left(\xi_{10}, \xi_{11}\right.$, and $\left.\xi_{12}\right)$ is unitary, and second, the matrix of the coefficients $Z_{i j}$, appearing in the expression for $p_{z}$ is symmetric and related to the zeta-matrix, $\zeta_{\alpha \beta}$ (i.e., the coefficients in the expression for $p_{z}$ in terms of normal coordinates), by the equation

$$
\zeta_{\alpha \beta}=\Sigma Z_{i j} S_{i \alpha} S_{i \beta}
$$

where the diagonal elements $\zeta_{\alpha \alpha}$ are the observable values $\zeta_{\alpha}=\zeta_{10}, \zeta_{11}$, and $\zeta_{12}$. The formal advantage of the use of a new set of coordinates $\xi_{i}{ }^{*}$ in accomplishing this same simplification for heavy ethane is offset by a consequent necessity for the introduction of a new set of potential constants $b_{i j}{ }^{*}$ related but not equal to the $b_{i j}$.
}

$$
\begin{aligned}
& S_{10,1}=0.636 S_{10,2}=0.630 S_{10,3}=0.446 / \xi_{10} \\
& \begin{array}{l}
S_{11,1}=-0.734 S_{11,2}=0.672 S_{11,3}=0.097 \\
S_{12,1}=0.239 S_{12,2}=0.389 S_{12,3}=-0.890
\end{array}\left(\begin{array}{l}
\xi_{10} \\
\xi_{11} \\
\xi_{12}
\end{array}\right)=S\left(\begin{array}{l}
\xi_{1} \\
\xi_{2} \\
\xi_{3}
\end{array}\right) \\
& S_{10,1}{ }^{*}=0.596 S_{10,2^{*}}=0.593 S_{10,3^{*}=}=0.542\left(\xi_{10^{*}}\right) \\
& S_{11,1^{*}}=-0.783 S_{11,2}{ }^{*}=0.578 S_{11,3^{*}}=0.228\left(\xi_{11}^{*}\right) \\
& S_{12,1^{*}}=0.178 S_{12,2^{*}}=0.560 S_{12,3}{ }^{*}=-0.809\left(\begin{array}{l}
\xi_{12}{ }^{*} \\
\xi^{*}
\end{array}\right) \\
& =S\left(\begin{array}{l}
\xi_{1} \\
\xi_{2} \\
\xi_{3} /(\sigma)^{\xi}
\end{array}\right)
\end{aligned}
$$

\section{Parallel Inactive Vibrations $\left(A_{1 g}\right)$}

The displacement $\xi_{1}$ is one in which each hydrogen atom moves outward away from the symmetry axis by an amount $\left[1 /(6)^{\frac{1}{2}}\right] \xi_{1}$. In the displacement $\xi_{2}$ each hydrogen triangle moves away from the molecule center by an amount $\left[1 /(6)^{\frac{1}{2}}\right] \xi_{2}$, and in the displacement $\xi_{3}$ each carbon atom moves towards the molecule center by an amount $(m / 2 M)^{\frac{1}{3}} \xi_{3}$. This choice of internal coordinates gives the following energy expressions:

$$
\begin{aligned}
T & =\frac{1}{2} m\left(\dot{\xi}_{1}{ }^{2}+\xi_{2}{ }^{2}+\dot{\xi}_{3}{ }^{2}\right) \\
T^{*} & =\frac{1}{2} m^{*}\left(\dot{\xi}_{1}{ }^{2}+\xi_{2}{ }^{2}+\xi_{3}{ }^{2} / \sigma\right) ; \quad \sigma=m^{*} / m \\
V & =V^{*}=\frac{1}{2} \Sigma c_{i j} \xi_{i} \xi_{j} .
\end{aligned}
$$

For the determination of the six potential constants, only five independent normal frequencies are available, and it is necessary to introduce one simplifying assumption. We have chosen equal to zero the valence force constant $k_{R r}$ which couples $\mathrm{C}-\mathrm{C}$ stretching to $\mathrm{C}-\mathrm{H}$ stretching, and the values of the potential constants $c_{i j}$ listed in Table VI are dependent upon this assumption.

The elements of the unitary matrices connecting the normal vibrations with the displacements $\xi_{1}, \xi_{2}$, and $\xi_{3}$ are

$\begin{array}{llll}S_{11}=0.980 & S_{12}=0.170 & S_{13}=0.102 \\ S_{21}=-0.198 & S_{22}=0.873 & S_{23}=0.445 \\ S_{31}=-0.013 & S_{32}=-0.456 & S_{33}=0.890\end{array}\left(\begin{array}{l}\xi_{1} \\ \xi_{2} \\ \xi_{3}\end{array}\right)=S\left(\begin{array}{l}\xi_{1} \\ \xi_{2} \\ \xi_{3}\end{array}\right)$

\section{Parallel Active Vibrations $\left(A_{2 u}\right)$}

In the displacement $\xi_{1}$, the hydrogen atoms of one triangle (the unprimed triangle) move outward while the atoms of the opposing triangle move inward towards the symmetry axis by the same amount $\left[1 /(6)^{\frac{1}{2}}\right] \xi_{1}$. In the displacement $\xi_{2}$, the hydrogen atoms move in the direction of the $-Z$ axis while the carbon atoms move in the direction of the $+Z$ axis by an amount such that there is no translation of the molecule center. The scale of $\xi_{2}$ is chosen such that the sum of the carbon and hydrogen (or deuterium) atom displacements is $(6 M / 3 m+M)^{\frac{1}{2}} \xi_{2}$ and this choice gives rise to the following energy expressions.

$$
\begin{aligned}
T & =\frac{1}{2} m\left(\dot{\xi}_{1}{ }^{2}+\dot{\xi}_{2}{ }^{2}\right) \\
T^{*} & =\frac{1}{2} m^{*}\left(\dot{\xi}_{1}{ }^{2}+\xi_{2}{ }^{2} / \sigma\right) ; \quad \sigma=\left(3 m^{*}+M\right) /(3 m+M) \\
V & =V^{*}=\frac{1}{2} \Sigma d_{i j} \xi_{i} \xi_{j}=\frac{1}{2}\left(d_{11} \xi_{1}{ }^{2}+d_{22} \xi_{2}{ }^{2}+2 d_{12} \xi_{1} \xi_{2}\right) .
\end{aligned}
$$


TABLE VII. Definition of valence force constants for ethane (excluding the force constant associated with torsion). $r_{i}$ denotes the $\mathrm{H}_{i}-\mathrm{C}$ distance minus the equilibrium distance, $R$ the $\mathrm{C}-\mathrm{C}$ distance minus the equilibrium distance, $\psi_{i}$ the increase in the $\mathrm{H}_{i}-\mathrm{C}-\mathrm{C}$ angle, $\theta_{i}$ the increase in the $\mathrm{H}_{i-1}-\mathrm{C}-\mathrm{H}_{i+1}$ angle.

\begin{tabular}{|c|c|c|}
\hline & Nature of the constant & Contribution to the potential energy \\
\hline \multirow[b]{2}{*}{$\begin{array}{l}(1) \\
(2) \\
(3) \\
(4)\end{array}$} & & (A) Stretching and bending force constants. \\
\hline & $\begin{array}{l}\mathrm{C}-\mathrm{H} \text { stretching } \\
\angle \mathrm{H}-\mathrm{C}-\mathrm{C} \text { bending } \\
\angle \mathrm{H}-\mathrm{C}-\mathrm{H} \text { bending } \\
\mathrm{C}-\mathrm{C} \text { stretching }\end{array}$ & 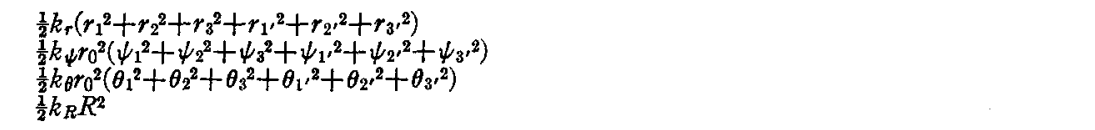 \\
\hline & \multirow{9}{*}{$\begin{array}{l}\mathrm{C}-\mathrm{H} \text { stretching } \\
\mathrm{C}-\mathrm{H} \text { stretching } \\
\mathrm{H}-\mathrm{C}-\mathrm{C} \text { bending } \\
\mathrm{H}-\mathrm{C}-\mathrm{C} \text { bending } \\
\mathrm{H}_{i}-\mathrm{C}-\mathrm{C} \text { bending } \\
\mathrm{H}_{i+1}-\mathrm{C}-\mathrm{H}_{i-1} \text { bending } \\
\mathrm{H}_{i}-\mathrm{C} \text { stretching } \\
\mathrm{H}_{i+1}-\mathrm{C}-\mathrm{C} \text { bending } \\
\mathrm{H}_{i}-\mathrm{C} \text { stretching } \\
\mathrm{H}_{i+1}-\mathrm{C}-\mathrm{H}_{i-1} \text { bending } \\
\mathrm{H}_{i}-\mathrm{C} \text { stretching } \\
\mathrm{H}_{i}-\mathrm{C}-\mathrm{C} \text { bending } \\
\mathrm{H}-\mathrm{C} \text { stretching } \\
\mathrm{C}-\mathrm{C} \text { stretching } \\
\mathrm{C}-\mathrm{C} \text { stretching } \\
\mathrm{H}-\mathrm{C}-\mathrm{C} \text { bending }\end{array}$} & Force constants coupling adjacent parts of the molecule. \\
\hline$(5)$ & & $\frac{1}{2} k_{r r}\left(r_{1} r_{2}+r_{2} r_{3}+r_{3} r_{1}+r_{1^{\prime}} r_{2^{\prime}}+r_{2} \cdot r_{3^{\prime}}+r_{3^{\prime}} r_{1^{\prime}}\right)$ \\
\hline (6) & & $\frac{1}{2} k_{\psi} \psi_{\psi} r_{0}^{2}\left(\psi_{1} \psi_{2}+\psi_{2} \psi_{3}+\psi_{3} \psi_{1}+\psi_{1^{\prime}} \psi_{2^{\prime}}+\psi_{2^{\prime}} \psi_{3^{\prime}}+\psi_{3^{\prime}} \psi_{1^{\prime}}\right)$ \\
\hline$(7)$ & & $\frac{1}{2} k_{\theta} \psi r_{0}^{2}\left(\theta_{1} \psi_{1}+\theta_{2} \psi_{2}+\theta_{3} \psi_{3}+\theta_{1^{\prime}} \psi_{1^{\prime}}+\theta_{2^{\prime}} \psi_{2^{\prime}}+\theta_{3^{\prime}} \psi_{3^{\prime}}\right)$ \\
\hline (8) & & $\frac{1}{4} k r \psi_{\beta} r_{0}\left\{r_{1}\left(\psi_{2}+\psi_{3}\right)+r_{2}\left(\psi_{3}+\psi_{1}\right)+r_{3}\left(\psi_{1}+\psi_{2}\right)+r_{1^{\prime}}\left(\psi_{2^{\prime}}+\psi_{3^{\prime}}\right)+r_{2}\left(\psi_{3^{\prime}}+\psi_{1^{\prime}}\right)+r_{3}\left(\psi_{1^{\prime}}+\psi_{2^{\prime}}\right)\right\}$ \\
\hline (9) & & $\frac{1}{2} k_{r} \theta r_{0}\left(r_{1} \theta_{1}+r_{2} \theta_{2}+r_{3} \theta_{3}+r_{1}, \theta_{1},+r_{2}, \theta_{2},+r_{3}, \theta_{3}\right)$ \\
\hline (10) & & $\frac{1}{2} k_{r} \psi_{0}\left(r_{1} \psi_{1}+r_{2} \psi_{2}+r_{3} \psi_{3}+r_{1}, \psi_{1^{\prime}}+r_{2^{\prime}} \psi_{2^{\prime}}+r_{3^{\prime}} \psi_{3^{\prime}}\right)$ \\
\hline (11) & & $\frac{1}{2} k_{r R} R\left(r_{1}+r_{2}+r_{3}+r_{1}+r_{2^{\prime}}+r_{3}\right)$ \\
\hline (12) & & $\frac{1}{2} k_{R \psi} r_{0} R\left(\psi_{1}+\psi_{2}+\psi_{3}+\psi_{1^{\prime}}+\psi_{2^{\prime}}+\psi_{3^{\prime}}\right)$ \\
\hline \multicolumn{2}{|r|}{ (C) } & Force constants coupling remote parts of the molecule. \\
\hline (13) & $\left\{\begin{array}{l}\mathbf{H}_{\mathbf{i}}-\mathrm{C} \text { stretching } \\
\mathbf{H}_{i}-\mathrm{C} \text { stretching }\end{array}\right.$ & $k_{r r^{\prime}}\left(r_{1} r_{1},+r_{2} r_{2}+r_{3} r_{3}\right)$ \\
\hline (14) & $\left\{\begin{array}{l}\mathrm{H}_{i}-\mathrm{C} \text { stretching } \\
\mathrm{C}-\mathrm{H}_{(i+1)} \text { stretching }\end{array}\right.$ & $\frac{1}{2} k r r^{\prime} \beta\left(r_{1} r_{2^{\prime}}+r_{1} r_{3^{\prime}}+r_{2} r_{1^{\prime}}+r_{2} r_{3^{\prime}}+r_{3} r_{1^{\prime}}+r_{3} r_{2^{\prime}}\right)$ \\
\hline (15) & $\left\{\begin{array}{l}\mathrm{H}_{i}-\mathrm{C} \text { stretching } \\
\mathrm{C}-\mathrm{C}-\mathrm{H}_{(i+1)}, \text { bending }\end{array}\right.$ & $\frac{1}{4} k r \psi^{\prime} \beta_{0}\left\{r_{1}\left(\psi_{2^{\prime}}+\psi_{3^{\prime}}\right)+r_{2}\left(\psi_{3^{\prime}}+\psi_{1^{\prime}}\right)+r_{3}\left(\psi_{1^{\prime}}+\psi_{2^{\prime}}\right)+r_{1^{\prime}}\left(\psi_{2}+\psi_{3}\right)+r_{2^{\prime}}\left(\psi_{3}+\psi_{1}\right)+r_{3^{\prime}}\left(\psi_{1}+\psi_{2}\right)\right\}$ \\
\hline (16) & $\left\{\begin{array}{l}\mathrm{H}_{i}-\mathrm{C} \text { stretching } \\
\mathrm{C}-\mathrm{C}-\mathrm{H}_{i}, \text { bending }\end{array}\right.$ & $\frac{1}{2} k_{r \psi^{\prime}} r_{0}\left(r_{1} \psi_{1^{\prime}}+r_{2} \psi_{2^{\prime}}+r_{3} \psi_{3^{\prime}}+r_{1^{\prime}} \psi_{1}+r_{2^{\prime}} \psi_{2}+r_{3^{\prime}} \psi_{3}\right)$ \\
\hline (17) & $\left\{\begin{array}{l}\mathrm{H}_{i}-\mathrm{C} \text { stretching } \\
\mathrm{H}_{(i+1)},-\mathrm{C}-\mathrm{H}_{(i-1)}, \text { bending }\end{array}\right.$ & $\frac{1}{2} k_{r \theta^{\prime}} r_{0}\left(r_{1} \theta_{1^{\prime}}+r_{2} \theta_{2^{\prime}}+r_{3} \theta_{3^{\prime}}+r_{1^{\prime}} \theta_{1}+r_{2^{\prime}} \theta_{2}+r_{3^{\prime}} \theta_{3}\right)$ \\
\hline (18) & $\left\{\begin{array}{l}\mathrm{H}_{i}-\mathrm{C}-\mathrm{C} \text { bending } \\
\mathrm{C}-\mathrm{C}-\mathrm{H}_{i}, \text { bending }\end{array}\right.$ & $k_{\psi} \psi^{\prime} r 0^{2}\left(\psi_{1} \psi_{1^{\prime}}+\psi_{2} \psi_{2^{\prime}}+\psi_{3} \psi_{3^{\prime}}\right)$ \\
\hline (19) & $\left\{\begin{array}{l}\mathrm{H}_{i}-\mathrm{C}-\mathrm{C} \text { bending } \\
\mathrm{C}-\mathrm{C}-\mathrm{H}_{(i+1)}, \text { bending }\end{array}\right.$ & $\frac{1}{2} k 44 \beta_{\beta^{\prime}} r_{0}^{2}\left(\psi_{1} \psi_{\Sigma^{\prime}}+\psi_{1} \psi_{3^{\prime}}+\psi_{2} \psi_{3^{\prime}}+\psi_{2} \psi_{1^{\prime}}+\psi_{3} \psi_{1^{\prime}}+\psi_{3} \psi_{2^{\prime}}\right)$ \\
\hline (20) & $\left\{\begin{array}{l}\mathrm{H}_{i+1}-\mathrm{C}-\mathrm{H}_{i-1} \text { bending } \\
\mathrm{H}_{(i+1)}, \mathrm{C}-\mathrm{H}_{(i-1)}, \text { bending }\end{array}\right.$ & $k_{\theta \theta^{\prime}} \cdot r_{0}^{2}\left(\theta_{1} \theta_{1^{\prime}}+\theta_{2} \theta_{2^{\prime}}+\theta_{3} \theta_{3^{\prime}}\right)$ \\
\hline (21) & $\left\{\begin{array}{l}\mathrm{H}_{i+1}-\mathrm{C}-\mathrm{H}_{i-1} \text { bending } \\
\mathrm{C}-\mathrm{C}-\mathrm{H}_{i^{\prime}} \text { bending }\end{array}\right.$ & $k_{\theta \psi^{\prime}} r_{0^{2}}^{2}\left(\theta_{1} \psi_{\mathbf{1}^{\prime}}+\theta_{2} \psi_{2^{\prime}}+\theta_{3} \psi_{3^{\prime}}\right)$ \\
\hline
\end{tabular}

The three independent normal frequencies associated with this symmetry class just suffice to determine the values of the $d_{i j}$ listed in Table VI.

The unitary matrices connecting the normal vibrations with the displacements $\xi_{1}$ and $\xi_{2}$ have the following numerical elements.

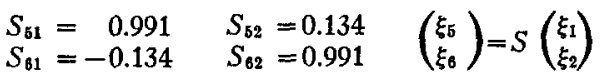

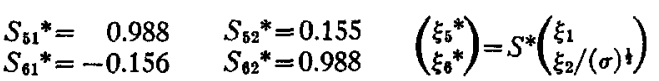

\section{E. The Torsional Vibration $\left(A_{1 u}\right)$}

In the displacement $\xi$ one hydrogen triangle rotates clockwise while the other rotates counterclockwaise about the symmetry axis, the displacement of any hydrogen atom being $\left[1 /(6)^{\prime}\right] \xi$. The energy expressions are thus

$$
\begin{aligned}
T & =\frac{1}{2} m \dot{\xi}^{2} \\
T^{*} & =\frac{1}{2} m^{*} \xi^{2} \\
V & =V^{*}=\frac{1}{2} e \xi^{2} .
\end{aligned}
$$

The torsional motion or hindered rotation in ethane represents a rather special case in which the shape of the potential energy function is approximately known over the whole range of the torsion angle $\theta$. Perhaps more significant than the potential constant $e$ is the value of the barrier height $V_{0}=965 \mathrm{~cm}^{-114}$ appearing in the assumed expression $V$ (torsion $)=V_{0}(1-\cos 3 \theta) / 2$.

\section{THE VALENCE FORCE CONSTANTS OF ETHANE}

By means of the general potential constants already evaluated, it is now possible to determine restoring forces associated with arbitrary small distortions of ethane such as the stretching of a $\mathrm{C}-\mathrm{H}$ bond or the bending of $\mathrm{H}-\mathrm{C}-\mathrm{H}$ angles. The force constants associated with such specific distortions are of importance mainly for comparisons with other molecules which possess similar components. Complicating such comparisons is the fact that when, for example, one of these bands is stretched, the remaining parts of the molecule deform in such a way as to minimize the poten- 
TABLE VIII. The valence force constants of ethane. (All constants are expressed in units of $10^{5}$ dynes $/ \mathrm{cm}$.)

Stretching and bending force constants

$\begin{array}{ll}\text { (1) } & k_{r}=5.35 \pm 0.03 \\ \text { (2) } & k_{\theta}=0.51 \pm 0.02 \\ \text { (3) } & k_{\psi}=0.63 \pm 0.02 \\ \text { (4) } & k_{R}=4.57 \pm 0.03\end{array}$

Coupling constants for adjacent components

$\begin{array}{ll}\text { (5) } & k_{r \psi \beta}=-1.89 \pm 0.20 \\ (6) & k_{R \psi}=0.60 \pm 0.04 \\ (7) & k_{r \theta}=-0.20 \pm 0.02 \\ (8) & k_{\theta \psi}=-0.07 \pm 0.02 \\ (9) & k_{r \psi}=-0.07 \pm 0.15 \\ (10) & k_{r r}=-0.04 \pm 0.04 \\ (11) & k_{\psi \psi}=0.03 \pm 0.04 \\ (12) & k_{R r}=0.00 \pm 0.20\end{array}$

Coupling constants for remote components

$\begin{array}{ll}(13) & k_{\psi \psi^{\prime}}=0.11 \pm 0.02 \\ (14) & k_{\theta \psi^{\prime}}=0.07 \pm 0.03 \\ (15) & k_{r \theta^{\prime}}=-0.03 \pm 0.02 \\ (16) & k_{\theta \theta^{\prime}}=-0.02 \pm 0.02 \\ (17) & k_{r r^{\prime} \beta}=-0.08 \pm 0.04 \\ (18) & k_{r r^{\prime}}=0.08 \pm 0.04 \\ (19) & k_{r \psi^{\prime} \beta}=0.35 \pm 0.20 \\ (20) & k_{r \psi^{\prime}}=-0.13 \pm 0.15 \\ (21) & k_{\psi \psi^{\prime} \beta}=0.00 \pm 0.05\end{array}$

tial energy. Such deformations are characterized by coupling force constants and indicate the strength of interaction between various parts of the molecule. It is well known, however, that the vibrational spectra of many molecules may be computed quite accurately by use of potential functions in which most of the possible coupling terms are assumed zero. One thus expects, $a$ priori, that many of the coupling terms for ethane will be small, especially those terms which represent coupling between the remote parts of the molecule.

These bond stretching, bond angle bending, and coupling force constants are the potential constants associated with a valence coordinate expression of the potential energy function. In the displacement corresponding to the torsional vibration, no bond distances and no bond angles are changed. Thus, on the assumption of pure valence forces, there would be no restoring force and this frequency would be zero. The observed value of the frequency is indeed rather small and the restoring force, which depends on its square, is considerably less than the force required to distort a bond angle. To this extent the measurements of the torsional frequency agree with the simplified valence picture. The remaining eleven frequencies all involve changes in bond distances and angles.

The nineteen bond distances and bond angles of ethane give rise to the nineteen valence coordinates having the following definitions:

$$
\begin{aligned}
& R=\text { increase in } \mathrm{C}-\mathrm{C} \text { distance (that is, the } \\
& \mathrm{C}-\mathrm{C} \text { separation minus the equilibrium } \\
& \text { separation) } \\
& \left.r_{i} \text { (or } r_{i^{\prime}}\right)=\text { the increase in } \mathrm{C}-\mathrm{H}_{i} \text { (or } \mathrm{C}-\mathrm{H}_{i^{\prime}} \text { ) } \\
& \text { distance, where the subscript } i \text { designates a } \\
& \text { particular hydrogen atom as labelled in } \\
& \text { Fig. } 8
\end{aligned}
$$

(8-13) $\psi_{i}=$ the increase in the $\mathrm{H}_{i}-\mathrm{C}-\mathrm{C}$ bond angle (14-19) $\theta_{i}=$ the increase in the $\mathrm{H}_{i-1}-\mathrm{C}-\mathrm{H}_{i+1}$ bond angle. These coordinates are not independent but satisfy the two conditions

$$
\begin{aligned}
& \text { (a) } \quad\left(\theta_{1}+\theta_{2}+\theta_{3}\right) \sin \theta_{0}=\left(\psi_{1}+\psi_{2}+\psi_{3}\right)\left(3 \sin \psi_{0} \cos \psi_{0}\right) \\
& \text { (b) }\left(\theta_{1^{\prime}}+\theta_{2^{\prime}}+\theta_{3^{\prime}}\right) \sin \theta_{0}=\left(\psi_{1^{\prime}}+\psi_{2^{\prime}}+\psi_{3^{\prime}}\right)\left(3 \sin \psi_{0} \cos \psi_{0}\right)
\end{aligned}
$$

which are equivalent to the conditions of no net rotation about the symmetry axis and no torsion of the methyl groups. Although the redundancy of the coordinate system permits a certain latitude in the expression of the potential energy, twenty-one independent force constants may be defined as in Table VII. As a consequence of the latitude in choice of the twenty-one independent quadratic forms constituting the expression

\begin{tabular}{|c|c|c|c|}
\hline & Nature of constant & Contribution to the potential energy & $\begin{array}{l}\text { Value of constant in } \\
\text { units of } 10^{5} \text { dynes } / \mathrm{cm}\end{array}$ \\
\hline (1) & $\mathrm{C}-\mathrm{H}$ stretching & $\frac{1}{2} K_{r}\left(r_{1}^{2}+r_{2}^{2}+r_{3}^{2}+r_{4}^{2}\right)$ & $K_{\mathrm{r}}=5.394$ \\
\hline (2) & $\angle \mathrm{H}-\mathrm{C}-\mathrm{H}$ bending & $\frac{1}{2} K_{\psi} r_{0}{ }^{2}\left(\psi_{12^{2}}+\psi_{13^{2}}{ }^{2}+\psi_{14}{ }^{2}+\psi_{23^{2}}{ }^{2}+\psi_{24}{ }^{2}+\psi_{34}{ }^{2}\right)$ & $K=0.420$ \\
\hline (3) & $\begin{array}{l}\mathrm{C}-\mathrm{H}_{i} \\
\mathrm{C}-\mathrm{H}_{i}\end{array}$ coupling & $\frac{1}{2} K_{r r}\left(r_{1} r_{2}+r_{2} r_{3}+r_{3} r_{4}+r_{4} r_{1}+r_{1} r_{3}+r_{2} r_{4}\right)$ & $K_{\mathrm{rr}}=0.037$ \\
\hline (4) & $\begin{array}{l}\mathrm{C}-\mathrm{H}_{i} \\
\mathrm{H}_{j}-\mathrm{C}-\mathrm{H}_{k} \text { coupling }\end{array}$ & $\frac{1}{2} K_{4} r_{0}\left\{r_{1}\left(\psi_{23}+\psi_{24}+\psi_{34}\right)+r_{2}\left(\psi_{13}+\psi_{14}+\psi_{34}\right)+r_{3}\left(\psi_{12}+\psi_{14}+\psi_{24}\right)+r_{4}\left(\psi_{12}+\psi_{13}+\psi_{23}\right)\right\}$ & $K_{4}=-0.276$ \\
\hline (5) & $\begin{array}{l}\mathrm{H}_{i}-\mathrm{C}-\mathrm{H}_{i} \\
\mathrm{H}_{k}-\mathrm{C}-\mathrm{H}_{\mathbf{l}}\end{array}$ & $\frac{1}{2} K_{5} r_{0}^{2}\left(\psi_{12} \psi_{34}+\psi_{13} \psi_{24}+\psi_{14} \psi_{23}\right)$ & $K_{5}=-0.075$ \\
\hline
\end{tabular}
of the potential function, the values of the angle bending force constants $k_{\theta}$ and $k_{\psi}$ depend on the particular expression of the coupling terms. This dependence, however, is quite weak due to the smallness of the coupling force constants. Table VIII gives the values of the valence force constants as obtained directly from the set

TABLE IX. Definition and values for the valence force constants of methane. $r_{i}$ denotes the $\mathrm{C}-\mathrm{H}_{i}$ distance minus the equilibrium distance, and $\psi_{i j}$ the increase in the $\mathrm{H}_{i}-\mathrm{C}-\mathrm{H}_{j}$ angle. 
TABLE X. Correlation of the valence force constants of ethane and methane.

(Force constants expressed in units of $10^{5}$ dynes $/ \mathrm{cm}$.)

\begin{tabular}{|c|c|c|c|}
\hline \multicolumn{2}{|c|}{ Nature of force constant } & Ethane & \multirow{2}{*}{$\begin{array}{l}\text { Methane } \\
\tau_{r}=5.394 \\
\psi=0.420 \\
=0.037 \\
r r=-0.276\end{array}$} \\
\hline $\begin{array}{l}\mathrm{C}-\mathrm{H} \text { stretching } \\
\mathrm{H}-\mathrm{C}-\mathrm{H} \text { bending } \\
\mathrm{C}-\mathrm{H}_{i} \text { to } \mathrm{C}-\mathrm{H}_{j} \text { cou } \\
\mathrm{C}-\mathrm{H}_{i} \text { to } \mathrm{H}_{i+1}-\mathrm{C}-\end{array}$ & ing & $\begin{array}{l}k_{r}=5.35 \\
k_{\theta}=0.51 \\
k_{r r}=-0.04 \\
k_{r \theta}=-0.20\end{array}$ & \\
\hline Fthe & \multicolumn{2}{|c|}{ Formal correlations } & Methane \\
\hline Nature of constant & Value & Value & Nature of constant \\
\hline $\begin{array}{l}\mathrm{C}-\mathrm{C} \text { stretching } \\
\mathrm{H}-\mathrm{C}-\mathrm{C} \text { bending } \\
\mathrm{C}-\mathrm{H} \text { to } \mathrm{C}-\mathrm{C} \text { coupling } \\
\mathrm{H}_{i-1}-\mathrm{C}-\mathrm{H}_{i+1} \text { to } \mathrm{H}_{i}-\mathrm{C}-\mathrm{C} \text { coupling } \\
\mathrm{C}-\mathrm{H}_{i} \text { to } \mathrm{H}_{i-1}-\mathrm{C}-\mathrm{C} \text { coupling } \\
\mathrm{C}-\mathrm{H}_{i} \text { to } \mathrm{H}_{i}-\mathrm{C}-\mathrm{C} \text { coupling } \\
\mathrm{C}-\mathrm{C} \text { to } \mathrm{H}_{i}-\mathrm{C}-\mathrm{C} \text { coupling } \\
\mathrm{H}_{i}-\mathrm{C}-\mathrm{C} \text { to } \mathrm{H}_{i-1}-\mathrm{C}-\mathrm{C} \text { coupling }\end{array}$ & $\begin{array}{l}k_{R}=4.57 \\
k_{\psi}=0.63 \\
k_{r R}=0.00 \\
k_{\theta \psi}=-0.07 \\
k_{r \psi \beta}=-1.99 \\
k_{r \psi}=-0.07 \\
k_{R \psi}=0.60 \\
k_{\psi \psi}=0.03\end{array}$ & $\begin{array}{l}K_{r}=5.394 \\
K_{\psi}=0.420 \\
K_{r r}=0.037 \\
K_{5}=-0.075 \\
2 K_{4}=-0.552 \\
" k_{r \psi}=0.000 \\
-K_{4}=0.276 \\
" k_{\psi \psi} \equiv 0.000\end{array}$ & $\begin{array}{l}\mathrm{C}-\mathrm{H} \text { stretching } \\
\mathrm{H}-\mathrm{C}-\mathrm{H} \text { bending } \\
\mathrm{C}-\mathrm{H}_{i} \text { to } \mathrm{C}-\mathrm{H}_{j} \text { coupling } \\
\mathrm{H}_{i-1}-\mathrm{C}-\mathrm{H}_{i+1} \text { to } \mathrm{H}_{i}-\mathrm{C}-\mathrm{H}_{i+2} \text { coupling } \\
\mathrm{C}-\mathrm{H}_{i} \text { to } \mathrm{H}_{i-1}-\mathrm{C}-\mathrm{H}_{i+1} \text { coupling } \\
\mathrm{C}-\mathrm{H}_{i} \text { to } \mathrm{H}_{i}-\mathrm{C}-\mathrm{H}_{i-1} \text { coupling } \\
\mathrm{C}-\mathrm{H}_{i} \text { to } \mathrm{H}_{i}-\mathrm{C}-\mathrm{H}_{i-2} \text { coupling } \\
\mathrm{H}_{i}-\mathrm{C}-\mathrm{H}_{i-2} \text { to } \mathrm{H}_{i-1}-\mathrm{C}-\mathrm{H}_{i-2} \text { coupling }\end{array}$ \\
\hline
\end{tabular}

of general potential constants associated with the previous symmetry coordinate system.

The "uncertainty values" shown in Table VIII are based on the changes in the computed force constants when the individual fundamental frequencies and zetavalues are given upper and lower limits consistent with the spectroscopic data (e.g., although the positions of the components of the resonance doublets $\nu_{1 a}$ and $\nu_{1 b}$ have been measured with high precision, the unperturbed value chosen for the fundamental frequency $\nu_{1}$ is necessarily only an estimate which may be in error by perhaps $\left.\pm 15 \mathrm{~cm}^{-1}\right)$. These "uncertainty values" are intended to give a measure of how well the present data determine the valence force constants and thus also the extent to which the values of the constants can be used for significant correlations with similar constants of other molecules.

It will be noticed that, for the most part, the principal contributions to the potential energy arise from changes in the bond distances and bond angles and not from the cross product terms. The potential energy associated with a change in a $\mathrm{C}-\mathrm{C}-\mathrm{H}$ angle is approximately but not exactly equal to that coming from a change in $\mathrm{H}-\mathrm{C}-\mathrm{H}$ angle. The coupling constants for adjacent components of the molecule are not very significantly different from zero with the exception of the interaction between a $\mathrm{C}-\mathrm{C}$ stretching and a $\mathrm{C}-\mathrm{C}-\mathrm{H}$ bending, a $\mathrm{H}_{i}-\mathrm{C}$ stretching and a $\mathrm{H}_{i+1}-\mathrm{C}-\mathrm{H}_{i-1}$ bending, and a $\mathrm{H}_{i}-\mathrm{C}$ stretching and a $\mathrm{H}_{i+1}-\mathrm{C}-\mathrm{C}$ bending. It seems very puzzling that these last two interactions should be so large.

\section{CORRELATION OF THE VALENCE FORCE CONSTANTS OF ETHANE AND METHANE}

The potential function for methane has been determined by Dennison ${ }^{6}$ and is characterized by a set of five potential constants. In terms of valence coordinates these constants may be given as in Table IX (the one relation among the six $\mathrm{H}_{i}-\mathrm{C}-\mathrm{H}_{j}$ angles, $\psi_{i j}$, again making possible a number of different explicit expressions for the potential energy). That the coupling terms for methane and ethane have been chosen in a manner appropriate for the direct comparison of the simple force constants can be verified by rewriting the potential function of methane treating the 4th hydrogen atom formally equivalent to the primed carbon (or primed methyl group) of ethane and then comparing the coefficients of the resulting quadratic terms with the corresponding force constants of ethane. The results of such a procedure are given in Table $\mathrm{X}$. The values of the principal force constants of both ethane and methane given here are in general higher than those cited for example in Herzberg which have been obtained by the use of fundamental rather than normal frequencies in the secular determinant.

It is a pleasure to acknowledge the many helpful discussions with Dr. L. G. Smith regarding the infrared spectrum of ethane. 